INTER NATIONAL MONETARY FUND
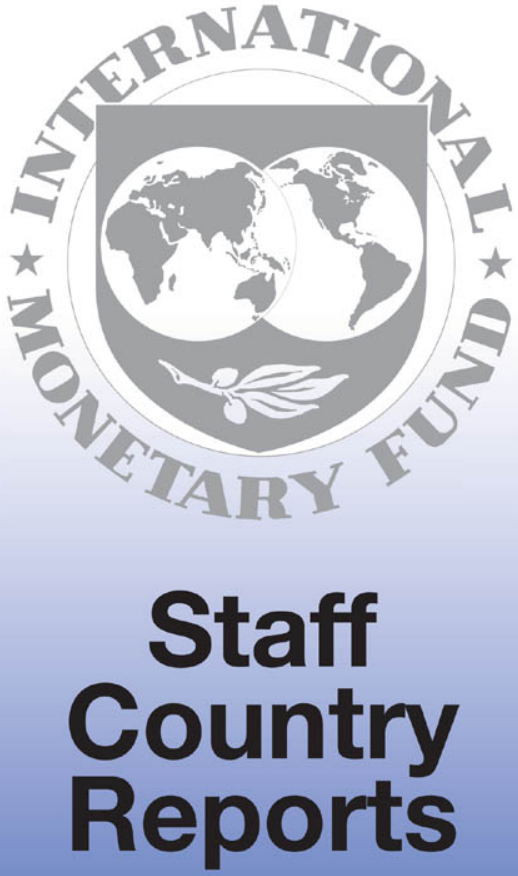


\section{Former Yugoslav Republic of Macedonia-Statistical Appendix}

This statistical appendix on the Former Yugoslav Republic of Macedonia was prepared by a staff team of the International Monetary Fund as background documentation for the periodic consultation with this member country. As such, the views expressed in this document are those of the staff team and do not necessarily reflect the views of the Government of the Former Yugoslav Republic of Macedonia or the Executive Board of the IMF.

Copies of this report are available to the public from

International Monetary Fund - Publication Services

700 19th Street, N.W. - Washington, D.C. 20431

Telephone: (202) 623-7430 - Telefax: (202) 623-7201

Telex (RCA): 248331 IMF UR

Internet: publications@imf.org

Price: $\$ 15.00$ a copy

International Monetary Fund

Washington, D.C. 
INTERNATIONAL MONETARY FUND

\title{
FORMER YUGOSLAV REPUBLIC OF MACEDONIA
}

\section{Statistical Appendix}

\author{
Prepared by Neven Mates and Vikram Haksar
}

Approved by the European I Department

March 7, 1997

Contents

Page

Tables

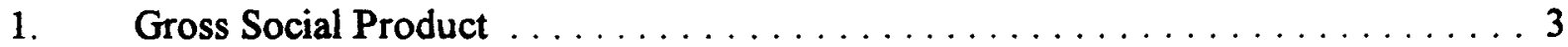

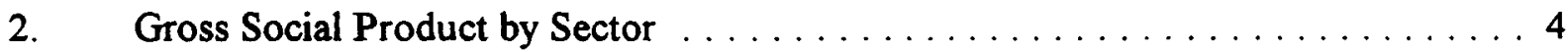

3. Gross Social Product and Aggregate Demand $\ldots \ldots \ldots \ldots \ldots \ldots \ldots \ldots \ldots$

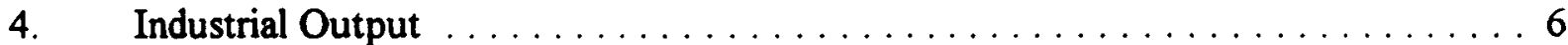

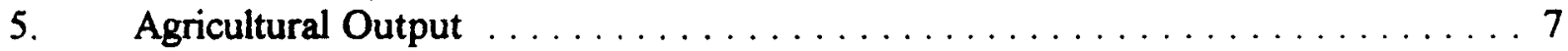

6. Primary Energy Production and Consumption $\ldots \ldots \ldots \ldots \ldots \ldots \ldots$

7. Secondary Energy Production and Consumption $\ldots \ldots \ldots \ldots \ldots \ldots \ldots$

8. Labor Force, Employment, and Unemployment $\ldots \ldots \ldots \ldots \ldots \ldots \ldots \ldots \ldots$

9. Output, Employment, Wages and Prices in Industry ............. 11

10. Nominal and Real Net Personal Income per Worker in the Enterprise Sector . . . 12

$11 . \quad$ Net Personal Income per Employee, by Sector ............... 13

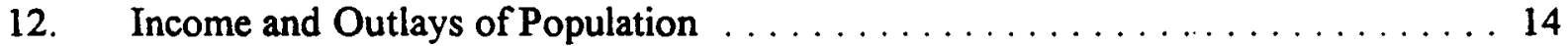

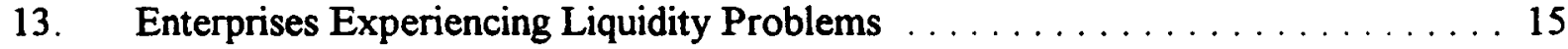

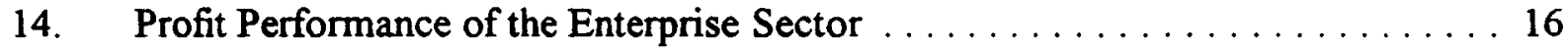

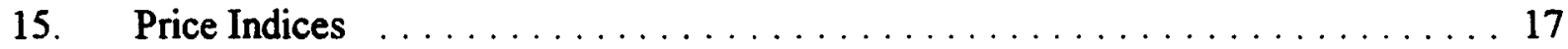

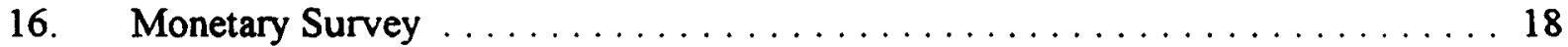

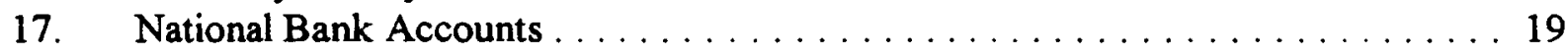

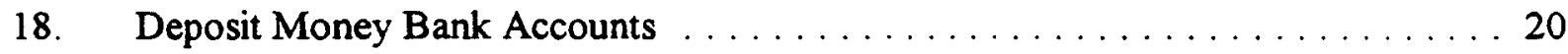

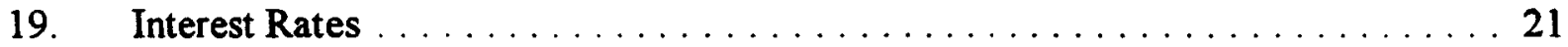

$20 . \quad$ Non-Government Sector Monetary Aggregates $\ldots \ldots \ldots \ldots \ldots \ldots \ldots \ldots 22$

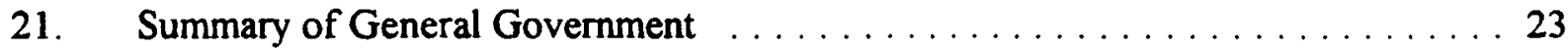

22. General Government Revenue and Grants $\ldots \ldots \ldots \ldots \ldots \ldots \ldots \ldots \ldots 24$

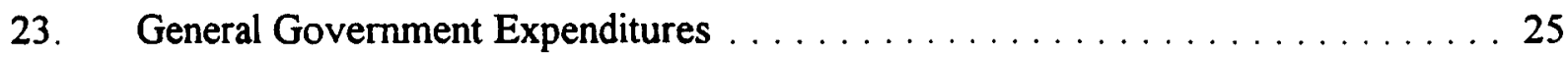

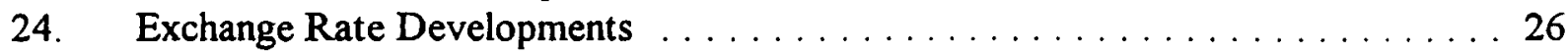

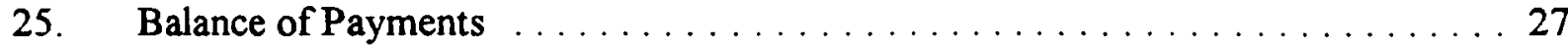

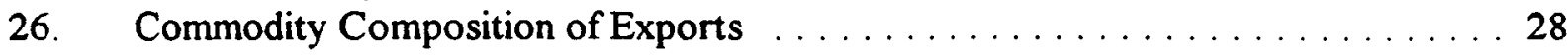


27. Destination of Exports . . . . . . . . . . . . . . . . . . . 29

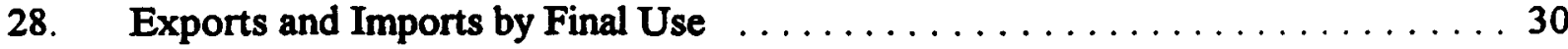

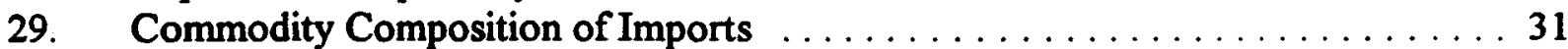

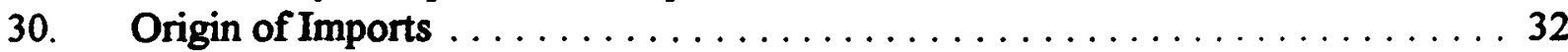

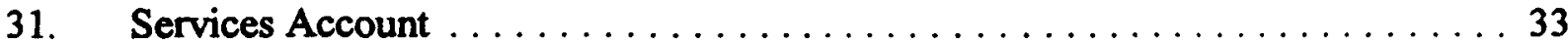

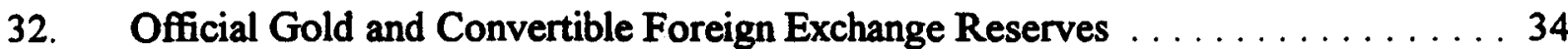

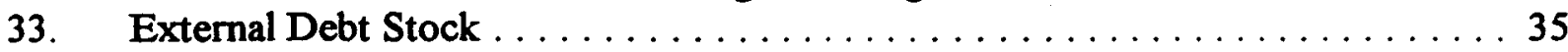


Table 1. FYRM: Gross Social Product

\begin{tabular}{|c|c|c|c|c|c|c|}
\hline & 1990 & 1991 & 1992 & 1993 & 1994 & $\frac{\text { Est. }}{1995}$ \\
\hline & \multicolumn{6}{|c|}{ GSP by Sector } \\
\hline & \multicolumn{6}{|c|}{ (share of GSP) } \\
\hline Manufacturing and mining & 48.0 & 45.6 & 44.7 & 44.4 & 42.8 & 39.3 \\
\hline Agriculture & 10.3 & 13.8 & 16.8 & 15.8 & 16.5 & 17.8 \\
\hline Construction & 7.3 & 6.6 & 6.6 & 6.8 & 6.2 & 6.6 \\
\hline Transport & 6.4 & 5.8 & 5.6 & 5.5 & 5.8 & 6.0 \\
\hline Trade & 19.8 & 19.3 & 18.0 & 18.4 & 18.1 & 19.7 \\
\hline Tourism & 1.9 & 1.8 & 1.9 & 2.5 & 2.2 & 2.0 \\
\hline Crafts & 1.8 & 2.0 & 1.9 & 2.1 & 2.0 & 2.1 \\
\hline Other & 4.6 & 5.1 & 4.5 & 4.4 & 6.4 & 6.6 \\
\hline \multirow[t]{2}{*}{ Total } & 100.0 & 100.0 & 100.0 & 100.0 & 100.0 & 100.0 \\
\hline & \multicolumn{6}{|c|}{ (Real percentage change) } \\
\hline Manufacturing and mining & -7.9 & -16.5 & -15.8 & -14.6 & -6.3 & -11.0 \\
\hline Agriculture & -17.4 & 17.6 & 4.8 & -19.1 & 1.6 & 4.0 \\
\hline Construction & -8.0 & -20.5 & -13.9 & -12.0 & -10.7 & 3.0 \\
\hline Transport & -15.0 & -20.0 & -17.7 & -15.0 & 2.7 & 0.0 \\
\hline Trade & -13.9 & -14.3 & -20.0 & -12.0 & -4.1 & 5.0 \\
\hline Tourism & -1.5 & -15.2 & -6.8 & 12.0 & -14.3 & -12.0 \\
\hline Crafts & -2.3 & 1.7 & -19.3 & -6.0 & -7.4 & 0.5 \\
\hline Other & 25.0 & -2.3 & -22.7 & -16.3 & 40.0 & 0.5 \\
\hline \multirow[t]{3}{*}{ GSP } & -9.7 & -12.1 & -14.0 & -14.1 & -2.6 & -3.2 \\
\hline & \multicolumn{6}{|c|}{ GSP based on aggregate demand } \\
\hline & \multicolumn{6}{|c|}{ (Contributions by component) } \\
\hline Private consumption & 57.4 & 54.0 & 51.0 & 55.5 & 57.1 & 57.2 \\
\hline Public consumption & 7.1 & 6.6 & 5.5 & 5.9 & 6.0 & 6.3 \\
\hline Gross investment & 19.3 & 18.6 & 17.0 & 15.0 & 16.0 & 17.4 \\
\hline Growth in stocks & 25.8 & 24.3 & 27.3 & 27.0 & 25.7 & 22.8 \\
\hline External balance & -9.6 & -3.6 & -0.7 & -3.4 & -4.8 & -3.7 \\
\hline \multirow[t]{2}{*}{ Total } & 100.0 & 100.0 & 100.0 & 100.0 & 100.0 & 100.0 \\
\hline & \multicolumn{6}{|c|}{ (Real percentage change) } \\
\hline Private consumption & -6.4 & -11.4 & -19.6 & -8.5 & -5.0 & -3.9 \\
\hline Public consumption & 12.7 & -12.5 & -30.1 & -8.9 & -6.9 & 1.4 \\
\hline Gross investment & 19.8 & -9.3 & -22.2 & -25.6 & -1.7 & 4.2 \\
\hline Growth in stocks & -15.0 & -11.1 & -4.6 & -16.6 & -12.3 & -14.9 \\
\hline External belance & -49.5 & 65.0 & 82.8 & -300.8 & -27.8 & 24.6 \\
\hline Total & -7.1 & -5.8 & -14.9 & -15.9 & -7.7 & -4.1 \\
\hline
\end{tabular}

Sources: The authorities; and staff estimates. 
Table 2. FYRM: Gross Social Product by Sector

\begin{tabular}{|c|c|c|c|c|c|c|}
\hline & 1990 & 1991 & 1992 & 1993 & 1994 & $\frac{\text { Prel }}{1995}$ \\
\hline & \multicolumn{6}{|c|}{ (In millions of current denars) } \\
\hline $\begin{array}{l}\text { Manufacture and mining: } \\
\text { Of which: }\end{array}$ & 238 & 451 & 7,155 & 28,228 & 56,572 & 63,569 \\
\hline Energy & 28 & 59 & 495 & 4,135 & $\ldots$ & $\ldots$ \\
\hline $\begin{array}{l}\text { Basic metals } \\
\text { Eouipment and }\end{array}$ & 29 & 65 & 781 & 2,830 & $\cdots$ & ... \\
\hline contruction durables & 38 & 70 & 1,214 & 4,217 & $\ldots$ & $\ldots$ \\
\hline construction materials & 19 & 47 & 611 & 2,312 & $\ldots$ & ... \\
\hline Chemicals & 21 & 39 & 653 & 1,126 & $\ldots$ & ... \\
\hline Other & 103 & 171 & 3,403 & 1,368 & $\ldots$ & ... \\
\hline Agriculture & 78 & 162 & 2,850 & 11,583 & 27,973 & 31,433 \\
\hline Construction & 44 & 81 & 1,056 & 5,007 & 9,473 & 10,645 \\
\hline Transport & 31 & 65 & 889 & 3,505 & 6,241 & 7,013 \\
\hline Trade & 109 & 280 & 2,878 & 15,521 & 31,956 & 35,909 \\
\hline Catering and tourism & 13 & 24 & 310 & 2,146 & 4,585 & 5,152 \\
\hline Crafts & 13 & 25 & 306 & 1,931 & 4,126 & 4,636 \\
\hline Other & 26 & 48 & 572 & 3,579 & 6,811 & 7,653 \\
\hline $\begin{array}{l}\text { Total GSP } \\
\text { Of which: }\end{array}$ & 551 & 1,137 & 16,016 & 71,500 & 147,737 & 166,010 \\
\hline Socialized sector & 472 & 947 & 12,847 & 49,891 & $\ldots$ & $\ldots$ \\
\hline \multirow[t]{2}{*}{ Private sector } & 79 & 189 & 3,169 & 10,520 & $\ldots$ & ... \\
\hline & & \multicolumn{5}{|c|}{ (In 1992 prices) } \\
\hline Manufacturing and mining & 10,177 & 8,498 & 7,155 & 6,110 & 5,726 & 5,096 \\
\hline Agriculture & 2,184 & 2,569 & 2,694 & 2,179 & 2,214 & 2,302 \\
\hline Construction & 1,542 & 1,226 & 1,056 & 929 & 830 & 854 \\
\hline Trancport & 1,350 & 1,080 & 889 & 756 & 776 & 776 \\
\hline Trade & 4,198 & 3,598 & 2,878 & 2,533 & 2,429 & 2,550 \\
\hline Catering and tourism & 393 & 333 & 310 & 347 & 298 & 262 \\
\hline Crafts & 373 & 379 & 306 & 287 & 266 & 267 \\
\hline Other & 964 & 942 & 728 & 610 & 853 & 857 \\
\hline Total GSP & 21,181 & 18,625 & 16,016 & 13,751 & 13,391 & 12,965 \\
\hline
\end{tabular}

Sources: The authorities; and staff estimates. 
Table 3. FYRM: Gross Social Product and Aggrogate Demand

(In millions of denars)

\begin{tabular}{|c|c|c|c|c|c|c|}
\hline & 1990 & 1991 & 1992 & 1993 & 1994 & ${ }_{19951 /}^{\text {Est. }}$ \\
\hline Private connumption & 289 & 601 & 7,920 & 37,682 & 75,930 & 84,701 \\
\hline Public consumption & 36 & 74 & 847 & 4,010 & 7,921 & 9,323 \\
\hline Groes invedument & 97 & 207 & 2,642 & 10,214 & 21,300 & 25,747 \\
\hline Growth in stocks & 130 & 271 & 4,240 & 18,376 & 34,178 & 33,759 \\
\hline External balance including formar Yugoalavia & -49 & -40 & -112 & $-2,334$ & $-6,328$ & $-5,538$ \\
\hline Commodity exports to other Yugonlov Republics & 0 & 11 & 1,334 & 4,620 & 5,921 & 6,977 \\
\hline Commodity imports from other Yugoulav Republics & $\mathbf{0}$ & 20 & 799 & 6,812 & 13,786 & 12,515 \\
\hline Interrepublicen trade balance & 0 & -9 & .535 & $-2,192$ & $-7,865$ & $-5,480$ \\
\hline \multicolumn{7}{|l|}{ Foreign belance } \\
\hline exchuding former Yugoslavia & -49 & -31 & -423 & -142 & 1,537 & $-3,983$ \\
\hline Exports of goods and services & 135 & 226 & 5,081 & 23,006 & 53,065 & 50,196 \\
\hline Goods & 126 & 216 & 4,770 & 20,249 & 41,539 & $\ldots$ \\
\hline Services & 9 & 10 & 311 & 2,758 & 11,256 & $\ldots$ \\
\hline Imports of goods and services & 183 & 256 & 5,503 & 23,148 & 51,528 & 54,179 \\
\hline Goods & 173 & 251 & 5,340 & 21,451 & 44,974 & $\ldots$ \\
\hline Services & 10 & 5 & 163 & 1,697 & 6,554 & $\ldots$ \\
\hline GSP demand side extimate & 504 & 1,114 & 15,537 & 67,948 & 133,001 & 147,992 \\
\hline Statistical discrepancy & 47 & 23 & 479 & 3,552 & 14,736 & 18,018 \\
\hline GSP & 551 & 1,137 & 16,016 & 71,500 & 147,737 & 166,010 \\
\hline \multicolumn{7}{|l|}{ Memcrondurn items: } \\
\hline \multicolumn{7}{|l|}{ Average exchenge rate } \\
\hline GSP implicit deflator & 100 & 235 & 3,846 & 19,998 & 42,433 & 49,246 \\
\hline \multicolumn{7}{|l|}{ Statistical discrepancy } \\
\hline as a percentage of demand side GSP & 9.3 & 2.0 & 3.1 & 5.2 & 11.1 & 12.2 \\
\hline as a percentage of GSP & 8.5 & 2.0 & 3.0 & 5.0 & 10.0 & 10.9 \\
\hline
\end{tabular}

Sources: Data provided by the authorities, and staff estimates.

1/ Ministry of Development estimates 
Table 4. FYRM: Industrial Output

\begin{tabular}{|c|c|c|c|c|c|c|}
\hline & 1991 & 1992 & 1993 & 1994 & 1995 & 1996 \\
\hline & \multicolumn{6}{|c|}{ (Index 1985=100) } \\
\hline Total industrial production & 83 & 70 & 61 & 54 & 48 & 50 \\
\hline Capital goods & 45 & 31 & 24 & 20 & 16 & $\ldots$ \\
\hline Intermediate goods & 82 & 70 & 63 & 55 & 52 & $\ldots$ \\
\hline Final goods & 97 & 83 & 70 & 68 & 61 & ... \\
\hline Electricity & 161 & 169 & 156 & 165 & 171 & 183 \\
\hline Petroleum products & 87 & 55 & 100 & 17 & 12 & $\ldots$ \\
\hline Iron and steel products & 85 & 54 & 37 & 21 & 22 & 32 \\
\hline Nonferrous metal products & 78 & 52 & 42 & 47 & 40 & 41 \\
\hline Nonmetal mineral products & 63 & 49 & 38 & 34 & 31 & 24 \\
\hline Electrieal machinery & 63 & 55 & 42 & 33 & 31 & 29 \\
\hline Basic chemical products & 59 & 47 & 38 & 37 & 33 & 30 \\
\hline Lumber industry & 60 & 51 & 46 & 42 & 30 & 28 \\
\hline Finished wood products industry & 85 & 67 & 45 & 38 & 26 & 19 \\
\hline Textile fiber and fabric industry & 61 & 50 & 44 & 41 & 31 & 29 \\
\hline Finished textile products industry & 85 & 69 & 69 & 58 & 50 & 44 \\
\hline Leather and fur industry & 78 & 68 & 53 & 48 & 44 & 53 \\
\hline \multicolumn{7}{|l|}{ Leather footwear and accessories } \\
\hline manufacturing & 80 & 73 & 45 & 31 & 22 & 22 \\
\hline & \multicolumn{6}{|c|}{ (Output of specific industrial products) } \\
\hline Electricity (MWHrs) & $5,770,137$ & $6,046,223$ & $5,069,136$ & $5,240,387$ & $5,331,950$ & ... \\
\hline Petroleum products ( $\mathrm{k}$ tons) & 884 & 556 & 987 & 161 & 113 & ... \\
\hline Sheet steel (tons) & 514,989 & $\ldots$ & 156,087 & 73,945 & 40,878 & $\ldots$ \\
\hline \multicolumn{7}{|l|}{ Buses-manufacture of } \\
\hline frames (tons) & 12,091 & 6,063 & 4,362 & 2,792 & 612 & $\ldots$ \\
\hline Agricultural machines (tons) & 521 & 301 & 295 & 222 & 67 & ... \\
\hline Refrigerators (units) & 135,972 & 138,937 & 98,478 & 95,192 & 56,148 & $\ldots$ \\
\hline Micro motors (KW) & 22,926 & 21,517 & 17,312 & 10,300 & 15,025 & $\cdots$ \\
\hline Cement (tons) & 605,735 & 516,053 & 499,093 & 486,450 & 523,499 & ... \\
\hline Wool yam (tons) & 8,089 & 7,007 & 5,858 & 4,856 & 3,863 & $\ldots$ \\
\hline Cotton fabric $(\mathbf{k ~ m 2})$ & 37,677 & 29,009 & 24,497 & 27,548 & 15,525 & ... \\
\hline Leather footwear ( $k$ pairs) & 3,566 & 3,303 & 2,076 & 1,510 & 1,120 & ... \\
\hline Cigarettes (millions) & 17,248 & 14,013 & 9,614 & 13,538 & 10,615 & ... \\
\hline
\end{tabular}

Sources: Statistical Office; and Ministry of Development. 
Table 5. FYRM: Agricultural Output

\begin{tabular}{|c|c|c|c|c|c|c|}
\hline & 1990 & 1991 & 1992 & 1993 & 1994 & 1995 \\
\hline & \multicolumn{6}{|c|}{ (In thousands of tons) } \\
\hline \multicolumn{7}{|l|}{$\begin{array}{l}\text { Production of selected } \\
\text { commodities }\end{array}$} \\
\hline Wheat & 231.4 & 340.7 & 299.5 & 249.8 & 336.1 & 381.2 \\
\hline Corn & 79.5 & 135.0 & 130.2 & 101.0 & 133.2 & 165.6 \\
\hline Sugar beets & 106.4 & 81.7 & 61.4 & 55.1 & 54.1 & 54.6 \\
\hline Sunflowers & 13.4 & 38.7 & 37.7 & 18.9 & 17.9 & 22.3 \\
\hline Tobacco & 16.4 & 25.2 & 26.5 & 21.1 & 18.9 & 15.7 \\
\hline Meat & 37.5 & 35.3 & 34.1 & 34.0 & 33.7 & 29.4 \\
\hline \multicolumn{7}{|l|}{ Of which: } \\
\hline Beef & 9.5 & 8.2 & 7.5 & 8.4 & 7.5 & 6.9 \\
\hline Pork & 9.7 & 9.9 & 10.4 & 9.6 & 9.7 & 9.3 \\
\hline Poultry & 2.3 & 2.4 & 2.3 & 1.9 & 2.1 & 1.9 \\
\hline Lamb/mutton & 14.4 & 13.1 & 12.4 & 12.9 & 13.0 & 10.0 \\
\hline Milk (million liters) & 179.9 & 179.5 & 175.8 & 178.0 & 177.1 & 197.8 \\
\hline & \multicolumn{6}{|c|}{ (Volume index 1985=100) } \\
\hline Total agricultural production: & 97.4 & 114.5 & 115.0 & 91.5 & 98.6 & 102.6 \\
\hline Socialized sector & 96.2 & 96.5 & 92.7 & 66.5 & 70.7 & 66.9 \\
\hline Private sector & 98.1 & 122.5 & 124.8 & 102.6 & 111.3 & 119.0 \\
\hline \multicolumn{7}{|l|}{$\begin{array}{l}\text { Production of selected } \\
\text { commodities: }\end{array}$} \\
\hline Wheat & 80.2 & 118.1 & 103.8 & 86.6 & 116.5 & 132.2 \\
\hline Corn & 100.4 & 170.5 & 164.4 & 127.5 & 168.2 & 209.1 \\
\hline Suear beets & 98.6 & 75.7 & 56.9 & 51.1 & 50.1 & 50.6 \\
\hline Sunflowers & 68.4 & 197.4 & 192.3 & 96.4 & 91.3 & 113.5 \\
\hline Tobecos & 53.4 & 82.1 & 86.3 & 68.7 & 61.4 & 51.0 \\
\hline $\begin{array}{l}\text { Meat } \\
\text { Of which: }\end{array}$ & 83.5 & 78.6 & 75.9 & 75.7 & 75.3 & 65.4 \\
\hline Beef & 86.3 & 74.5 & 68.1 & 76.4 & 68.8 & 62.9 \\
\hline Pork & 80.8 & 82.5 & 86.7 & 80.0 & 80.2 & 77.5 \\
\hline Poultry & 30.3 & 31.6 & 30.3 & 25.0 & 28.1 & 24.6 \\
\hline Lamb/mutton & 120.0 & 109.2 & 103.3 & 107.5 & 108.3 & 82.8 \\
\hline \multirow[t]{2}{*}{ Milk (million liters) } & 117.5 & 117.2 & 114.8 & 116.3 & 115.7 & 129.2 \\
\hline & \multicolumn{6}{|c|}{ (Share of agricultural production) } \\
\hline Socialized sector & 28.6 & 25.4 & 24.3 & 21.9 & 23.1 & 20.8 \\
\hline Private sector & 71.4 & 74.6 & 75.7 & 78.1 & 76.9 & 79.2 \\
\hline
\end{tabular}

Sources: Data provided by the Statistical Office; and Ministry of Development. 
Table 6. FYRM: Primary Energy Production and Consumption 1/

\begin{tabular}{|c|c|c|c|c|c|c|}
\hline & Units & 1991 & 1992 & 1993 & 1994 & 1995 \\
\hline Coal (including coking coal) & 1000 tons & & & & & \\
\hline Production & & 6,922 & 6,494 & 7,205 & 6,830 & 6,550 \\
\hline Imports & & 230 & 225 & 303 & 238 & 222 \\
\hline Exports & & 150 & 35 & 20 & 30 & 0 \\
\hline Consumption & & 7,002 & 6,684 & 7,488 & 7,068 & 6,772 \\
\hline Crude petroleum & 1000 tons & & & & & \\
\hline Production & & $\ldots$ & $\ldots$ & $\ldots$ & $\ldots$ & $\ldots$ \\
\hline Imports & & 920 & 760 & 900 & 142 & $\ldots$ \\
\hline Available for refining & & 920 & 760 & 900 & 142 & ... \\
\hline Hydroelectric power & mill. KwH & & & & & \\
\hline Potential & & 870 & 834 & 730 & 670 & 818 \\
\hline \multicolumn{7}{|l|}{ Total primary energy } \\
\hline Production 2/ & & 56.9 & 53.7 & 58.6 & 53.6 & 49.7 \\
\hline Imports 2/ & & 42.2 & 35.4 & 42.7 & 10.4 & 4.2 \\
\hline Exports 2/ & & 1.2 & 0.3 & 0.2 & 0.2 & 0.0 \\
\hline Consumption $2 /$ & & 97.9 & 88.8 & 100.8 & 63.8 & 53.9 \\
\hline
\end{tabular}

Source: Data provided by the authorities.

1/ Consumption encompasses all forms of use, including further transformation, additions to stocks, and non-energy uses.

2/ Thousands of terajoules $\left(10^{15}\right.$ joules). 
Table 7. FYRM: Secondary Energy Production and Consumption

\begin{tabular}{|c|c|c|c|c|c|}
\hline & 1991 & 1992 & 1993 & 1994 & 1995 \\
\hline & \multicolumn{5}{|c|}{ (In thousands of terajoules) 1/ } \\
\hline \multicolumn{6}{|l|}{$\begin{array}{l}\text { Electric energy } \\
\text { (hydro and thermal) }\end{array}$} \\
\hline Production & 23.1 & 20.1 & 22.2 & 19.6 & 20.4 \\
\hline Imports & 1.1 & 1.5 & 1.7 & 0.9 & 0.7 \\
\hline Exports & 1.5 & 1.0 & 0.0 & 0.5 & 0.2 \\
\hline Consumption & 22.2 & 20.7 & 23.9 & 20.0 & 20.9 \\
\hline \multicolumn{6}{|l|}{ Petroleum products } \\
\hline Production & 40.4 & 20.9 & 42.7 & 6.0 & 0.0 \\
\hline Imports & 7.7 & 15.0 & 9.9 & 34.4 & 32.7 \\
\hline Exports & 4.4 & 0.0 & 0.0 & 0.0 & 0.0 \\
\hline Consumption & 43.7 & 35.9 & 52.6 & 40.4 & 32.7 \\
\hline \multicolumn{6}{|l|}{ Coke } \\
\hline Production & 0.0 & 0.0 & 0.0 & 0.0 & 0.0 \\
\hline Imports & 2.8 & 3.0 & 2.6 & 2.2 & 2.1 \\
\hline Consumption & 2.8 & 3.0 & 2.6 & 2.2 & 2.1 \\
\hline \multicolumn{6}{|l|}{ Total secondary energy } \\
\hline Production & 63.5 & 41.0 & 64.9 & 25.6 & 20.4 \\
\hline Imports & 11.6 & 19.5 & 14.2 & 37.5 & 35.5 \\
\hline Exports & 5.9 & 1.0 & 0.0 & 0.5 & 0.2 \\
\hline Consumption & 68.7 & 59.6 & 79.1 & 62.6 & 35.7 \\
\hline \multicolumn{6}{|l|}{ Momorndum item: } \\
\hline Energy imports/consumption $2 /$ & 1.0 & 0.9 & 0.8 & 0.8 & 0.7 \\
\hline
\end{tabular}

Source: Data provided by the authorities.

$1 / 10^{15}$ joules.

2/ Primary plus secondary energy imports relative to secondary energy consumption. 
Table 8. FYRM: Labor Force, Employment, and Unemployment

(Anouil merom)

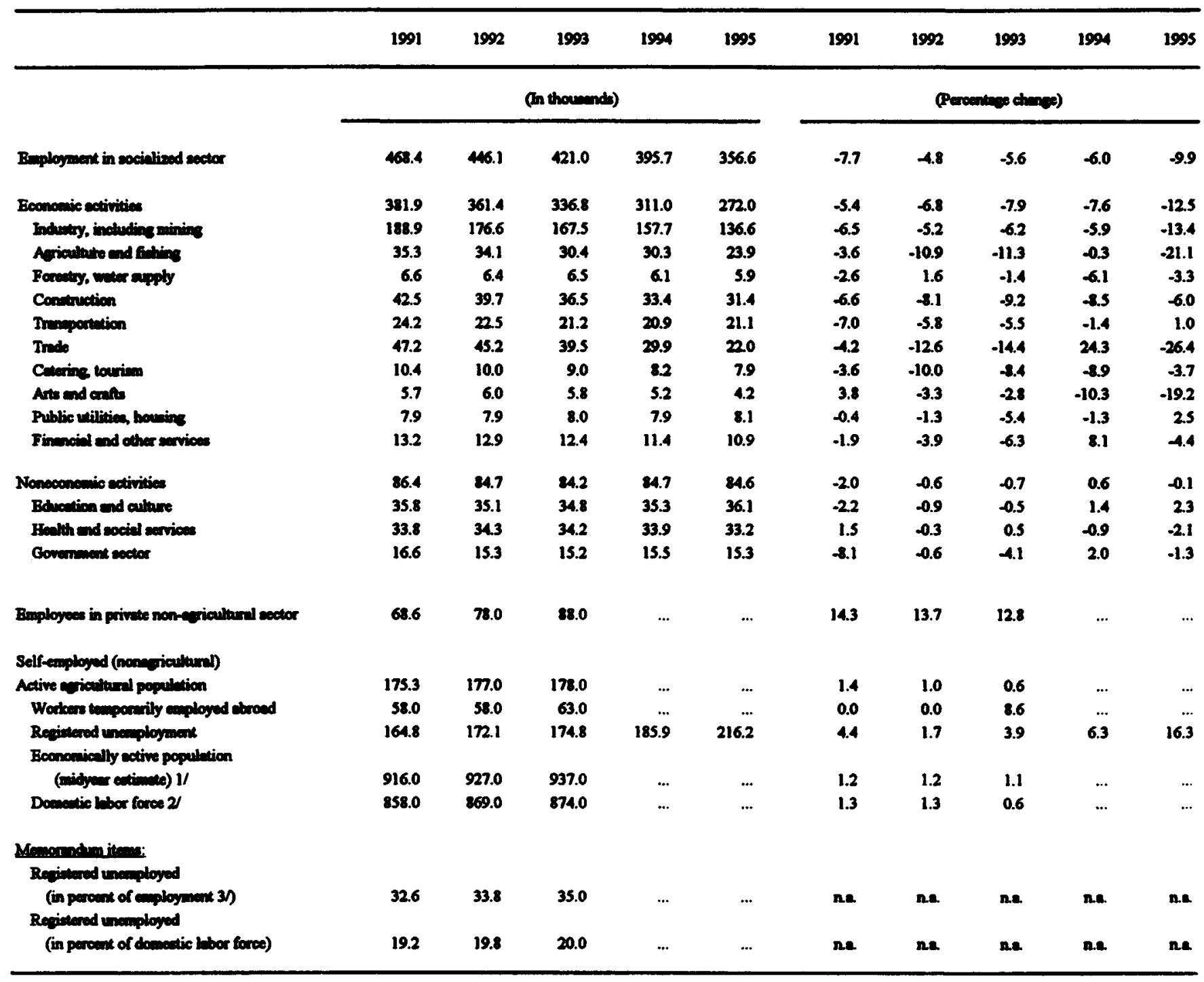

Source: Dun providad by the withoritien

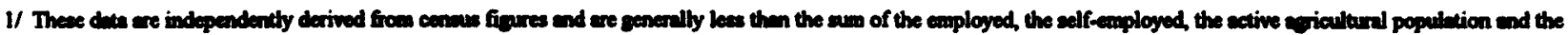

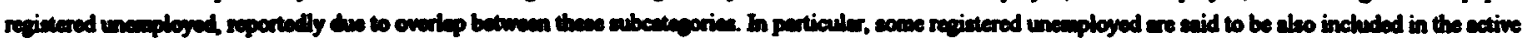
viculand popilation or the privite sector.

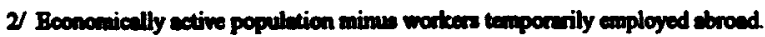

3/ In pareent of the amo of social end privise secter employment. 
Table 9. FYRM: Output, Employment, Wages and Prices in Industry

(Annual percent change)

\begin{tabular}{lcccccc}
\hline & 1991 & 1992 & 1993 & 1994 & 1995 & $19962 /$ \\
\hline $\begin{array}{l}\text { Industrial production 1/ } \\
\text { Employment }\end{array}$ & -17.2 & -15.9 & -15.0 & -10.5 & -10.7 & 3.4 \\
$\begin{array}{l}\text { Labor productivity } \\
\text { Net persomal income } \\
\text { per employee }\end{array}$ & -6.5 & -5.2 & -6.2 & -5.9 & -13.4 & -5.7 \\
$\begin{array}{l}\text { Industrial producer prices } \\
\text { M }\end{array}$ & -11.4 & -11.3 & -9.4 & -4.9 & 3.1 & 9.6 \\
\hline
\end{tabular}

Sources: Data provided by the authorities.

1/ In constant prices.

2/ Based in part on staff estimates. 
Table 10. FYRM: Nominal and Real Net Personal Income Per Worker in the Enterprise Sector 1/

\begin{tabular}{|c|c|c|c|c|}
\hline & \multicolumn{2}{|c|}{ Net personal income per worker } & \multicolumn{2}{|c|}{ Real net personal income per worker } \\
\hline & $\begin{array}{l}\text { Denar per } \\
\text { month }\end{array}$ & $\begin{array}{c}\text { Percent } \\
\text { change 2/ }\end{array}$ & $\begin{array}{c}\text { Index } \\
1985=100\end{array}$ & $\begin{array}{l}\text { Percent } \\
\text { change } 2 /\end{array}$ \\
\hline 1991 & 5,877 & 84.3 & 83.2 & -12.6 \\
\hline 1992 & 63,544 & 981.3 & 55.9 & -32.9 \\
\hline $19933 /$ & 3,782 & 495.6 & 72.0 & 28.9 \\
\hline 1994 & 7,668 & 102.7 & 65.2 & -9.4 \\
\hline 1995 & 8,581 & 11.9 & 61.8 & -4.3 \\
\hline 1996 & 8,822 & 2.8 & 61.0 & -1.3 \\
\hline \multicolumn{5}{|l|}{1995} \\
\hline Jamuary & 8,444 & 25.9 & 60.5 & -6.6 \\
\hline February & 8,351 & 17.3 & 59.8 & -6.4 \\
\hline March & 8,460 & 13.0 & 59.7 & -9.3 \\
\hline April & 8,472 & 12.7 & 59.6 & -7.5 \\
\hline May & 8,752 & 12.6 & 61.5 & -5.6 \\
\hline June & 8,625 & 9.9 & 62.4 & -1.6 \\
\hline July & 8,546 & 8.1 & 62.2 & -3.9 \\
\hline August & 8,739 & 8.9 & 63.6 & -2.8 \\
\hline September & 8,760 & 8.3 & 63.3 & -1.4 \\
\hline October & 8,755 & 6.0 & 61.2 & -4.2 \\
\hline November & 8,649 & 6.7 & 59.3 & -3.5 \\
\hline December & 8,435 & 0.1 & 57.3 & -8.0 \\
\hline \multicolumn{5}{|l|}{1996} \\
\hline January & 8,560 & 1.4 & 58.0 & -3.9 \\
\hline February & 8,572 & 2.6 & 58.0 & -2.7 \\
\hline March & 8,581 & 1.4 & 58.4 & -2.1 \\
\hline April & 8,695 & 2.6 & 59.5 & -0.1 \\
\hline May & 8,979 & 2.6 & 59.5 & -0.1 \\
\hline June & 8,762 & 1.6 & 61.6 & -1.3 \\
\hline July & 8,865 & 3.7 & 63.1 & 1.5 \\
\hline August & 8,959 & 2.5 & 64.5 & 1.4 \\
\hline September & 8,874 & 1.3 & 63.6 & 0.4 \\
\hline October & 9,065 & 3.5 & 62.7 & 2.4 \\
\hline November & 9,015 & 4.2 & 61.7 & 4.0 \\
\hline December & 8,931 & 5.9 & 61.1 & 6.7 \\
\hline
\end{tabular}

Source: Data provided by the Statistical Office.

1/ Actual take home pay of employees.

2/ Change from corresponding period of preceding year.

3/ On May 15, 1993, denar was re-denominated and 100 denars was equal to 1 new denar. 
Table 11. FYRM: Net Personal Income per Employee, By Sector 1/

\begin{tabular}{|c|c|c|c|c|c|c|c|c|c|c|}
\hline & 1991 & 1992 & 1993 & 1994 & 1995 & 1991 & 1992 & 1993 & 1994 & 1995 \\
\hline & \multicolumn{5}{|c|}{ (Denars per month) } & \multicolumn{5}{|c|}{ (Annual percentage change) } \\
\hline Total socialized sector & 59 & 635 & 3,782 & 7,754 & 8,581 & 84.3 & 981.2 & 495.6 & 105.0 & 10.7 \\
\hline $\begin{array}{l}\text { Economic sector } \\
\text { of which: }\end{array}$ & 55 & 607 & 7,341 & 7,391 & 8,302 & 79.4 & $1,009.5$ & 478.9 & 110.3 & 12.3 \\
\hline Industry (including mining) & 55 & 654 & 3,623 & 7,456 & 8,286 & 79.2 & $1,084.1$ & 454.0 & 105.8 & 11.1 \\
\hline Agriculture & 45 & 522 & 2,782 & 6,022 & 6,876 & 70.4 & $1,055.7$ & 440.2 & 116.5 & 14.2 \\
\hline Construction & 47 & 499 & 2,716 & 5,727 & 5,388 & 54.7 & 961.1 & 444.3 & 110.9 & 11.5 \\
\hline Transport & 64 & 659 & 4,272 & 8,816 & 9,604 & 87.6 & 923.9 & 548.3 & 106.4 & 8.9 \\
\hline Trade & 58 & 554 & 3,118 & 7,454 & 9,034 & 93.2 & 862.8 & 462.8 & 139.1 & 21.2 \\
\hline Catering, tourism & 44 & 487 & 3,114 & 6,568 & 6,948 & 69.5 & $1,001.9$ & 539.4 & 110.9 & 5.8 \\
\hline Handicrafts & 59 & 574 & 3,296 & 6,751 & 7,710 & 84.8 & 879.8 & 474.2 & 103.7 & 14.8 \\
\hline Public utilities, housing & 62 & 532 & 3,937 & 8,082 & 9,361 & 105.1 & 760.0 & 640.0 & 105.3 & 15.8 \\
\hline Financial, other services & 77 & 759 & 5,768 & 12,407 & 13,228 & 109.2 & 880.0 & 659.9 & 115.1 & 6.6 \\
\hline Noneconomic sector & 78 & 764 & 4,854 & 9,009 & 9,373 & 102.0 & 881.9 & 535.3 & 85.6 & 4.0 \\
\hline Education and culture & 72 & 749 & 4,866 & 8,841 & 9,217 & 94.0 & 934.1 & 549.7 & 81.7 & 4.3 \\
\hline Health, social security & 79 & 756 & 4,753 & 8,945 & 9,333 & 104.7 & 834.8 & 528.7 & 88.2 & 4.3 \\
\hline Government and other & 86 & 817 & 5,067 & 9,478 & 9,760 & 113.5 & 846.4 & 520.2 & 87.1 & 3.0 \\
\hline \multicolumn{11}{|l|}{ Memonndum item: } \\
\hline $\begin{array}{l}\text { Cost of living } \\
\text { (percentage change) }\end{array}$ & $\ldots$ & $\ldots$ & $\ldots$ & $\cdots$ & $\ldots$ & 110.8 & $1,511.3$ & 362.0 & 128.3 & 15.7 \\
\hline
\end{tabular}

Sources: Data provided by Statistical Office; and Ministry of Development.

1/ Actual take home pay of employees. 
Table 12. FYRM: Income and Outlaye of Population

\begin{tabular}{|c|c|c|c|c|c|}
\hline & 1990 & 1991 & $19921 /$ & $19931 /$ & $19941 /$ \\
\hline & \multicolumn{5}{|c|}{ (In millions of now denar) } \\
\hline Total incosie of population & 428 & 814 & 10,470 & 54,652 & 118,200 \\
\hline 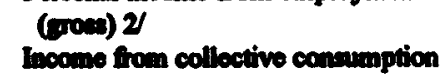 & 332 & 675 & 6,985 & 34,285 & 71,200 \\
\hline Punds & 6 & 10 & 0.0 & 0.0 & 0.0 \\
\hline Net inswest income & 11 & 32 & 1,300 & 4,000 & 5,000 \\
\hline Social security related transfors & 62 & 98 & 1,240 & 9,142 & 22,100 \\
\hline Tranafers from abroed, net & 17 & -2 & 486 & 5,450 & 15,800 \\
\hline Discropancios & 1 & 1 & 459 & 1,775 & 4,100 \\
\hline Taxes and contributions & 8 & 33 & 180 & 2,555 & 14,725 \\
\hline Disposeble income & 420 & 781 & 10,290 & 52,097 & 103,475 \\
\hline \multirow[t]{2}{*}{$\begin{array}{l}\text { Coanmption 3/ } \\
\text { Saving 4 } \\
\text { Housing, other fixed invotments } \\
\text { Finmoial savings S/ } \\
\text { Diecrepencios }\end{array}$} & $\begin{array}{r}289 \\
114 \\
41 \\
73 \\
17\end{array}$ & $\begin{array}{r}590 \\
158 \\
82 \\
76 \\
34\end{array}$ & $\begin{array}{r}7,920 \\
1,700 \\
660 \\
0.0 \\
670\end{array}$ & $\begin{array}{r}37,682 \\
8,700 \\
5,200 \\
3,500 \\
5,715\end{array}$ & $\begin{array}{r}75,970 \\
17,295 \\
10,700 \\
6,595 \\
10,210\end{array}$ \\
\hline & \multicolumn{5}{|c|}{ (In percent of total income) } \\
\hline Total income of population & 100 & 100 & 100 & 100 & 100 \\
\hline $\begin{array}{l}\text { Perwonal income from employment } \\
\text { (eros) } 2 / \\
\text { Income from colloctive consumption }\end{array}$ & 78 & 83 & 67 & 63 & 60 \\
\hline Fuada & 1 & 1 & 0.0 & 0.0 & 0.0 \\
\hline Net interest income & 3 & 4 & 12 & 7 & 4 \\
\hline Social sourity related tranufers & 14 & 12 & 12 & 17 & 19 \\
\hline Tranafers from shroad, not & 4 & $\mathbf{0}$ & 5 & 10 & 13 \\
\hline Discrepancies & $\mathbf{0}$ & $\mathbf{0}$ & 4 & 3 & 4 \\
\hline Troses and contributions & 2 & 4 & 2 & 5 & 13 \\
\hline Disposable income & 98 & 96 & 98 & 95 & 88 \\
\hline Consumption 3/ & 68 & 73 & 76 & 69 & 64 \\
\hline Seving 4 & 27 & 19 & 16 & 16 & 15 \\
\hline Housing, other fixed investunents & 10 & 10 & 6 & 10 & 9 \\
\hline Financial savines 5/ & 17 & 9 & 0.0 & 6 & 6 \\
\hline Discrepancies & 4 & 4 & 6 & 11 & 9 \\
\hline \multicolumn{6}{|l|}{ Momornnduen items: } \\
\hline $\begin{array}{l}\text { Cont of living index }(1988-100) \\
\text { Real personal net income from }\end{array}$ & 9,505 & 20,037 & 322,852 & $1,491,578$ & $3,405,273$ \\
\hline onployment $(1988=100)$ & 104 & 91 & 61 & 79 & 71 \\
\hline
\end{tabular}

Sources: Statistical Office; and Ministry of Dovelopment; and stuff estimates.

1/ Calculated in the Ministry of Development by using its methodology difierent then the Statisticel Ofifice's 2) The dat "income from collective consumption" are included in "gross personal income from employment for 1992 cawards.

$3 /$ Comamption is calculated as a residual.

4 Savings from 1993 and 1994 include intorests from previous savings, as thoy are treated as deferred savings.

5/ Exclusive of revaluation of foreign exchange eccounts. 
Table 13. FYRM: Enterprises Experiencing Liquidity Problems

(End-of-period)

1990

June

September

December

1991

\section{Mrroh}

June

September

October

1992

\section{March}

June

September

December

1993

\section{March}

June

September

December

1994

March

June

September

December

1995

March

June

September

December

1996
March

June

September
2,875
95,593

101,822

91,311

Sources: Data provided by the Payments Operation Service; and Ministry of Development.

1/ Those enterprises whose accounts with the Payments Operation Service do not contain sufificient funds to cover payment obligations as at the last day of the period. 
Table 14. FYRM: Profit Performance of the Enterprise Sector (In percent of GDP)

\section{Lossmakers}

Operating loss

4.6

Net financing costs of lossmakers

8.1

1.7

Loss after financing costs

12.6

9.7

Profitable firms

Operating profit

Net financing costs of profitable firms

Profit after financing costs

Extraordinary items

Net profit before tax

Depreciation

Net operating surplus

Memorandum items:

Gross wage arrears

Gross wage arrears in lossmaking firms

Special restructuring program

Net loss before tax in 113 SRP units

Number of units having loss

$7.3 \quad 1.9$
0

Gross wage arrears

as percent of wage costs

... 68

Source: Enterprise income statements as reported by Payment Operations System (POS). 
Table 15. FYRM: Price Indices

(Percent change)

\begin{tabular}{|c|c|c|c|c|c|c|c|c|}
\hline & & \multicolumn{2}{|c|}{ Retail Frices } & \multicolumn{2}{|c|}{$\begin{array}{c}\text { Indudrial } \\
\text { Produce Price }\end{array}$} & \multicolumn{2}{|c|}{$\begin{array}{l}\text { Ayroulumal } \\
\text { Producos Price }\end{array}$} & \multirow{2}{*}{$\begin{array}{c}\text { CSP } \\
\text { Defintor } \\
\text { Amenal } \\
\text { Crowith }\end{array}$} \\
\hline & & $\begin{array}{l}12 \text { mouth } \\
\text { Change } 1 /\end{array}$ & $\begin{array}{l}\text { Mouthly } \\
\text { Change 2/ }\end{array}$ & $\begin{array}{l}\text { 12-month } \\
\text { Chanpe } 1 /\end{array}$ & $\begin{array}{l}\text { Mouthly } \\
\text { Chnege } 2 /\end{array}$ & $\begin{array}{l}\text { 12-mowth } \\
\text { Change } 1 /\end{array}$ & $\begin{array}{l}\text { Mouthly } \\
\text { Change 2/ }\end{array}$ & \\
\hline 1990 & & 120.5 & 608.4 & 41.8 & 393.8 & 103.8 & 644.4 & 382.0 \\
\hline 1991 & & 229.7 & 114.9 & 281.5 & 112.0 & 182.9 & 67.2 & 129.3 \\
\hline 1992 & & $1,925.2$ & $1,690.7$ & $2,148.6$ & $2,198.2$ & $1,858.1$ & $1,698.0$ & $1,610.5$ \\
\hline 1993 & & 229.6 & 349.8 & 176.1 & 258.3 & 191.9 & 273.9 & 425.2 \\
\hline 1994 & & 55.4 & 121.8 & 28.6 & 88.9 & 14.6 & 16.0 & 123.2 \\
\hline 1995 & & 9.3 & 16.4 & 2.2 & 4.9 & 14.3 & 16.8 & $15.93 /$ \\
\hline 1996 & & 0.2 & 3.1 & -0.6 & -0.1 & $\ldots$ & $\ldots$ & 5.831 \\
\hline 1994 & Jamery & 244.1 & 22.0 & 177.2 & 13.1 & 108.9 & 13.8 & $\cdots$ \\
\hline & Fobrany & 178.9 & 6.8 & 118.2 & 3.9 & 72.9 & 5.8 & $\ldots$ \\
\hline & Mexch & 162.7 & 2.4 & 117.7 & 2.6 & 79.1 & 9.7 & $\ldots$ \\
\hline & April & 160.1 & 2.4 & 104.4 & 1.0 & 12.1 & -3.0 & $\ldots$ \\
\hline & May & 144.8 & 1.6 & 109.1 & 3.5 & 108.6 & 25.9 & .. \\
\hline & Juno & 153.0 & 2.3 & 106.4 & 0.5 & 200.4 & -12.2 & $\ldots$ \\
\hline & July & 133.6 & -0.6 & 99.1 & 0.0 & 120.9 & -21.4 & .. \\
\hline & Anguat & 115.4 & 0.6 & 91.3 & 0.8 & 120.0 & -13.5 & $\ldots$ \\
\hline & Sepplember & 105.8 & 1.8 & 84.6 & 0.0 & 93.4 & -2.9 & $\ldots$ \\
\hline & Octaber & 87.1 & 2.0 & 67.8 & 0.5 & 55.6 & 3.8 & ... \\
\hline & November & 71.3 & 2.2 & 53.0 & 1.0 & 61.9 & 15.9 & $\ldots$ \\
\hline & Decomber & 53.4 & 2.9 & 28.6 & -0.8 & 3.9 & -7.7 & $\ldots$ \\
\hline 1995 & January & 31.2 & 3.2 & 14.3 & 0.6 & -4.4 & 4.8 & $\ldots$ \\
\hline & Pobmary & 23.0 & 0.2 & 11.4 & 1.2 & -21.0 & -12.5 & $\ldots$ \\
\hline & March & 22.1 & 1.6 & 8.9 & 0.3 & -6.9 & 29.1 & $\ldots$ \\
\hline & April & 20.0 & 0.6 & 7.1 & -0.7 & -7.4 & -3.5 & $\ldots$ \\
\hline & May & 17.0 & -0.9 & 3.6 & 0.2 & 160.0 & 253.6 & $\ldots$ \\
\hline & June & 12.4 & -1.7 & 3.0 & -0.1 & 41.1 & -52.4 & $\ldots$ \\
\hline & July & 13.1 & 0.0 & 3.0 & 0.0 & 12.7 & -37.2 & $\ldots$ \\
\hline & Anguat & 12.6 & 0.2 & -0.1 & -2.3 & 3.2 & -20.8 & $\ldots$ \\
\hline & Seplomber & 11.5 & 0.7 & 2.0 & 2.2 & 9.7 & 3.2 & $\ldots$ \\
\hline & October & 12.5 & 3.0 & 2.1 & 0.5 & 0.5 & -4.8 & ... \\
\hline & November & 11.9 & 1.6 & 1.0 & 0.0 & -4.8 & 9.8 & $\ldots$ \\
\hline & December & 9.3 & 0.5 & 2.2 & 0.3 & 8.9 & 5.6 & $\ldots$ \\
\hline 1996 & Jenuary & 6.2 & 0.3 & 1.9 & 0.3 & 5.1 & 1.1 & $\ldots$ \\
\hline & February & 6.0 & 0.0 & 0.0 & -0.7 & 12.1 & -6.7 & $\ldots$ \\
\hline & Merch & 4.3 & 0.0 & 0.4 & 0.7 & 2.6 & 18.2 & $\ldots$ \\
\hline & April & 3.0 & -0.7 & -0.4 & -1.4 & 6.0 & -0.3 & $\ldots$ \\
\hline & May & 2.8 & -1.1 & 0.0 & 0.5 & 19.9 & 299.9 & $\ldots$ \\
\hline & June & 4.1 & -0.4 & -0.4 & -0.4 & -5.9 & -62.6 & $\ldots$ \\
\hline & July & 3.5 & -0.6 & -0.8 & -0.5 & -9.8 & -39.8 & $\ldots$ \\
\hline & Auguad & 2.5 & -0.7 & 1.3 & -0.1 & 3.1 & -9.5 & $\ldots$ \\
\hline & September & 2.1 & 0.3 & -1.7 & -0.9 & $\ldots$ & ... & $\ldots$ \\
\hline & October & 1.7 & 2.6 & -0.1 & 2.1 & $\ldots$ & $\ldots$ & $\ldots$ \\
\hline & Novennber & 0.8 & 0.7 & -0.2 & -0.1 & $\ldots$ & $\ldots$ & ... \\
\hline & December & 0.2 & -0.1 & -0.6 & -0.1 & ... & $\ldots$ & $\ldots$ \\
\hline
\end{tabular}

Sources: National Bark, Statiatical Office and Ministry of Development

1/ Amoual figures refer to Decumber-on-December inflation.

2/ Anmual figunes refer to annual average inflation.

3/ Preliminary extimates. 
Table 16. FYRM: Mancury survey $1 /$

(In mivion of domath ad of peciod)

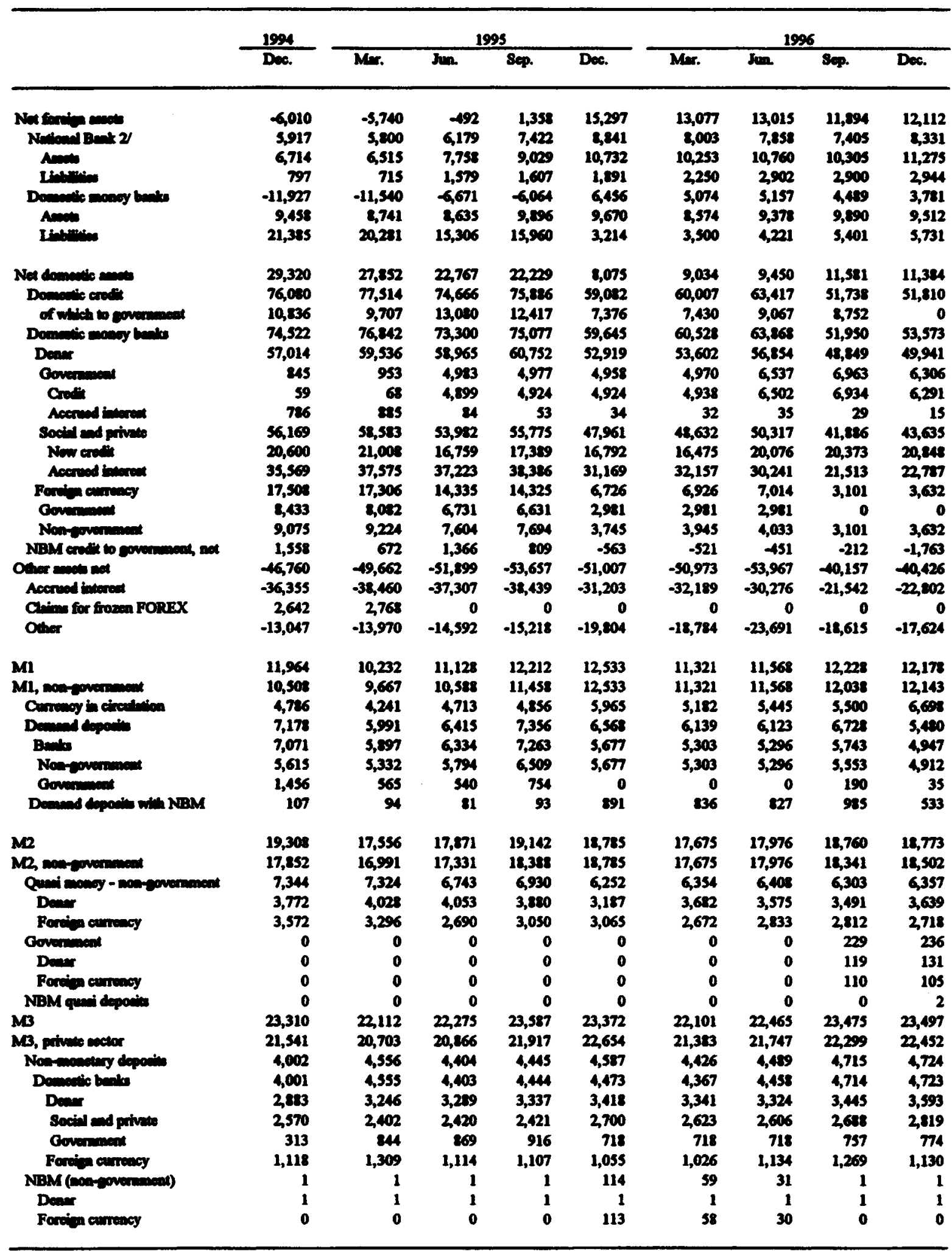

Source: Naional Bunk.

1/ A reported by the Recourch Department of the NBM, at current exchange rates. Exchudes frozen forcien currency deposits.

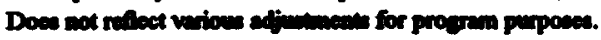

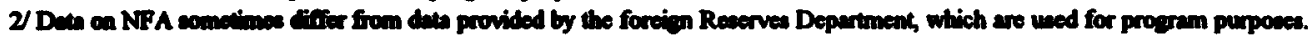


Table 17. FYRM: National Bank Accounts 1/

(In million of denars)

\begin{tabular}{|c|c|c|c|c|c|c|c|c|c|c|}
\hline \multirow[t]{2}{*}{ Period Ending } & \multirow[t]{2}{*}{1993} & \multirow[t]{2}{*}{1994} & \multicolumn{4}{|c|}{1995} & \multicolumn{4}{|c|}{1996} \\
\hline & & & Mar. & Jun. & Sep. & Dec. & Mar. & Jun. & Sep. & Dec. \\
\hline Net foreign assets & 5,513 & 5,917 & 5,800 & 6,179 & 7,422 & 8,841 & 8,003 & 7,858 & 7,405 & 8,331 \\
\hline Net domestic assets & $-1,751$ & -415 & $-1,294$ & $-1,299$ & $-2,367$ & $-2,571$ & $-2,617$ & $-2,170$ & $-1,586$ & $-1,216$ \\
\hline Banks & 1,275 & 1,262 & 982 & 370 & -84 & 2,690 & 2,628 & 3,022 & 3,501 & 4,799 \\
\hline Credits & 1,275 & 2,021 & 2,461 & 2,067 & 1,726 & 4,045 & 4,235 & 4,314 & 4,505 & 5,360 \\
\hline Instruments $2 /$ & -129 & -759 & $-1,479$ & $-1,697$ & $-1,810$ & $-1,355$ & $-1,607$ & $-1,292$ & $-1,004$ & -561 \\
\hline Government & 683 & 1,558 & 672 & 1,366 & 809 & -563 & -521 & -451 & -212 & $-1,763$ \\
\hline Credit & 684 & 1,621 & 1,723 & 2,708 & 2,703 & 1,860 & 1,860 & 1,857 & 1,850 & 1,848 \\
\hline Deposit & -1 & -63 & $-1,072$ & $-1,874$ & $-1,929$ & $-2,083$ & $-2,438$ & $-2,742$ & $-3,084$ & $-3,828$ \\
\hline Credit for external operations & $\ldots$ & $\mathbf{0}$ & 21 & 532 & 35 & -340 & 57 & 434 & 1,022 & 217 \\
\hline Other, net $3 /$ & 557 & $-3,235$ & $-2,948$ & $-3,035$ & $-3,092$ & $-4,698$ & $-4,724$ & $-4,741$ & $-4,875$ & $-4,252$ \\
\hline Reserve money & 3,382 & 5,502 & 4,506 & 4,880 & 5,054 & 6,271 & 5,386 & 5,688 & 5,819 & 7,115 \\
\hline Currency & 2,825 & 4,786 & 4,241 & 4,713 & 4,856 & 5,965 & 5,182 & 5,445 & 5,500 & 6,698 \\
\hline Other $4 /$ & 557 & 716 & 265 & 167 & 198 & 306 & 204 & 243 & 319 & 417 \\
\hline
\end{tabular}

Sources: Data provided by the authorities.

1/ At current exchange rates.

2/ Mandatory reserves and holdings of National Bank instruments.

3/ Includes non-financial sector deposits with the NBM.

4/ Vault cash and banks' excess reserves. 
Table 18. FYRM: Deposit Money Bank Accounts 1 1

(In million denars)

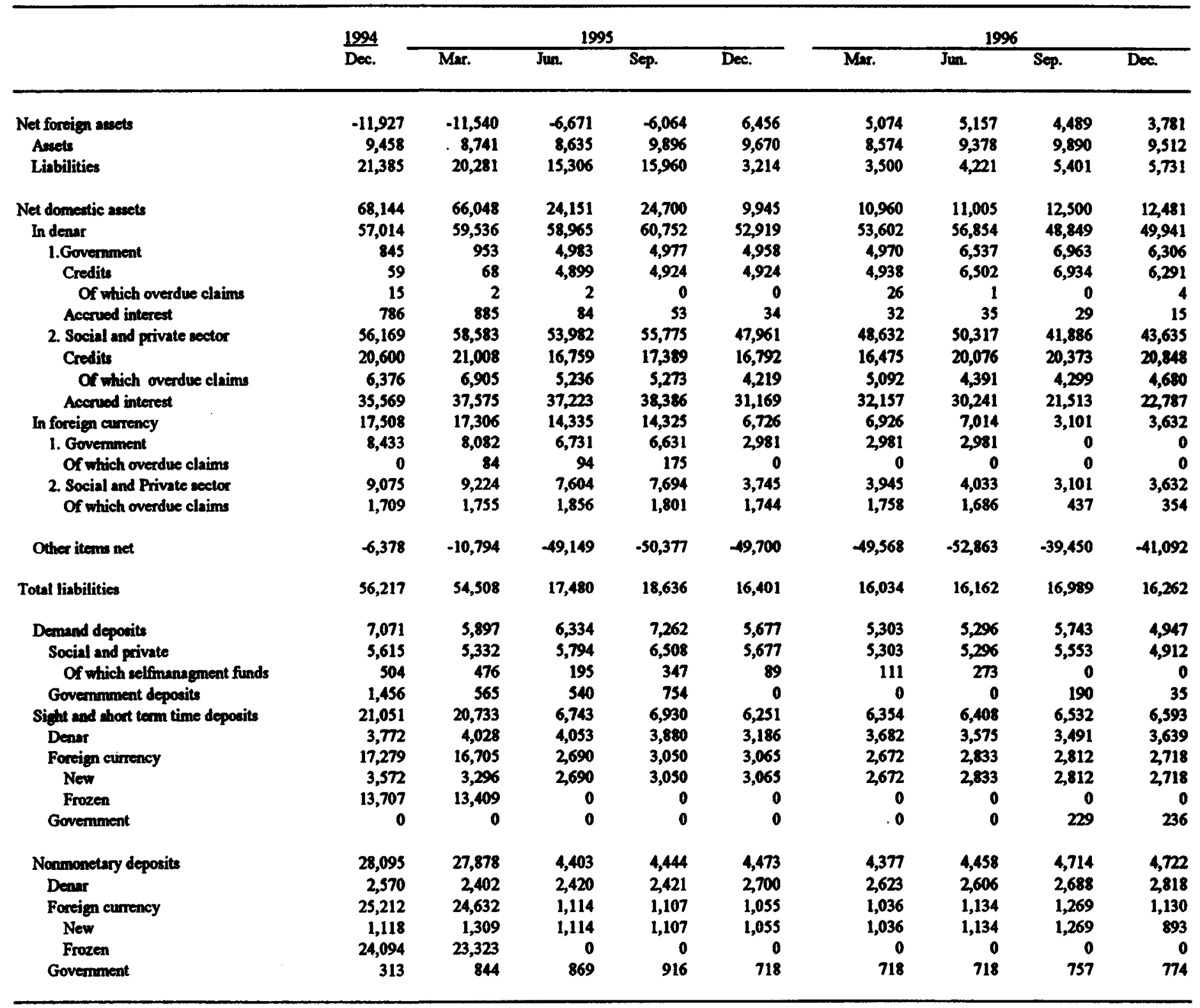

Source: National Bank

1/ In current exchange rates. Includes frozen foreign currency deposits until June 1995.

CInternational Monetary Fund. Not for Redistribution 
Table 19. FYRM: Interest Rates

(Annual)

\begin{tabular}{|c|c|c|c|c|c|c|c|c|c|c|c|}
\hline & \multirow{2}{*}{$\frac{1992}{\text { Dec. }}$} & \multirow{2}{*}{$\frac{1993}{\text { Dec. }}$} & \multirow{2}{*}{$\frac{1994}{\text { Dec. }}$} & \multicolumn{4}{|c|}{1995} & \multicolumn{4}{|c|}{1996} \\
\hline & & & & Mar. & Jun. & Sep. & Dec. & Mar. & Jun. & Sep. & Dec. \\
\hline \multicolumn{12}{|l|}{ NBM interest rates: } \\
\hline Discount rate & 250.0 & 295.0 & 33.0 & 28.0 & 18.0 & 18.0 & 15.0 & 12.0 & 10.0 & 9.2 & 9.2 \\
\hline Interest on regular liquidity credit & 719.0 & 848.0 & 66.0 & 56.0 & 36.0 & 36.0 & 30.0 & 24.0 & 20.0 & 18.4 & 18.4 \\
\hline Default interest rate & 813.0 & 959.0 & 99.0 & 84.0 & 54.0 & 54.0 & 45.0 & 36.0 & 30.0 & 27.6 & 27.6 \\
\hline \multicolumn{12}{|l|}{ Interest on required reserves } \\
\hline - sight dep. up to 3 years & 75.0 & 89.0 & 12.8 & 10.8 & 6.9 & 6.9 & 5.8 & 4.6 & 3.9 & 3.6 & 3.6 \\
\hline - over 3 years & 175.0 & 207.0 & 12.8 & 10.8 & 6.9 & 6.9 & 5.8 & 4.6 & 3.9 & 3.6 & 3.6 \\
\hline \multicolumn{12}{|l|}{ Commercial bank interest rates: } \\
\hline \multicolumn{12}{|l|}{ Deposit rates } \\
\hline Household sight dep. & $81-152$ & $50-78$ & $6-13$ & $5-13$ & 4-5 & 4-5 & $2-5$ & $7-8$ & $3-8$ & $3-7$ & 3-6 \\
\hline Household time dep. (3-6 months) & $435-885$ & $322-418$ & $32-49$ & $28-49$ & $18-21$ & $18-22$ & $9-21$ & $9-21$ & $9-21$ & $9-21$ & $9-21$ \\
\hline Enterprises sight dep. & $61-152$ & 42-78 & $6-13$ & $5-13$ & $3-12$ & 4-12 & $2-5$ & $2-10$ & $2-7$ & 4-7 & 4-7 \\
\hline Enterprises time dep. (3-6 months) & 435-885 & $207-418$ & $33-49$ & $34-49$ & $14-21$ & $14-22$ & $9-21$ & $10-21$ & $8-21$ & $8-21$ & $5-21$ \\
\hline \multicolumn{12}{|l|}{ Lending rates: } \\
\hline Short-term agricultural loans & $1,200-1,220$ & $275-675$ & 77.93 & $59-93$ & 30.0 & 30.0 & $\cdots$ & $\cdots$ & $\ldots$ & $\ldots$ & $\cdots$ \\
\hline Short-term consumer credit & $380-1,100$ & 191447 & $81-97$ & $59-98$ & 30.0 & 30.0 & $\ldots$ & $\ldots$ & $\ldots$ & $\ldots$ & $\ldots$ \\
\hline 'Short-term rates & $\ldots$ & $\ldots$ & $\ldots$ & $\ldots$ & $\ldots$ & $\ldots$ & $25-30$ & $20-30$ & $19-30$ & $19-30$ & $19-30$ \\
\hline Long-term working capital & $1,100-2,355$ & $474-675$ & $88-98$ & $59-92$ & 30.0 & 30.0 & $25-30$ & $19-30$ & $19-30$ & $19-30$ & $19-30$ \\
\hline Long-term housing credit & $310-1,686$ & $191-602$ & $81-162$ & $50-99$ & 30.0 & 30.0 & $25-30$ & $\ldots$ & $\ldots$ & $\ldots$ & $\ldots$ \\
\hline Small-scale industry & 1,100 & $367-602$ & $87-93$ & $77-93$ & 30.0 & 30.0 & $25-30$ & $25-35$ & $20-30$ & $20-30$ & $20-30$ \\
\hline
\end{tabular}

Source: Data provided by the National Bank. 
Table 20. FYRM: Non-government Sector Monetary Aggregates

(In million of denars, end of period)

\begin{tabular}{|c|c|c|c|c|c|c|c|c|c|}
\hline & Denar M1 & $\begin{array}{c}\text { Currency } \\
\text { in } \\
\text { Circulation }\end{array}$ & $\begin{array}{l}\text { Demand } \\
\text { Deposits }\end{array}$ & M2 & $\begin{array}{l}\text { Denar } \\
\text { Saving and } \\
\text { Time } \\
\text { Deposits } \\
\end{array}$ & $\begin{array}{c}\text { Foreign } \\
\text { Currency } \\
\text { Sight } \\
\text { Deposits }\end{array}$ & M3 & $\begin{array}{c}\text { Denar } \\
\text { non-monetary } \\
\text { Deposits }\end{array}$ & $\begin{array}{l}\text { Foreign } \\
\text { Currency } \\
\text { Time } \\
\text { Deposits } \\
\end{array}$ \\
\hline $1992 \mathrm{Q4}$ & 1,661 & 893 & 768 & 2,049 & 388 & 0 & 2,204 & 155 & $\ldots$ \\
\hline $1993 \mathrm{Q} 1$ & 2,049 & 908 & 1,141 & 2,810 & 641 & 120 & 3,064 & 254 & $\ldots$ \\
\hline $\mathbf{Q} 2$ & 3,093 & 1,480 & 1,613 & 4,979 & 1,308 & 578 & 5,397 & 418 & $\ldots$ \\
\hline Q3 & 4,306 & 1,980 & 2,326 & 7,559 & 2,302 & 951 & 8,241 & 682 & $\ldots$ \\
\hline Q4 & 5,591 & 2,703 & 2,888 & 13,539 & 3,560 & 4,388 & 15,142 & 1,603 & $\ldots$ \\
\hline 1994 Q1 & 6,296 & 2,715 & 3,581 & 15,279 & 4,596 & 4,387 & 16,889 & 1,610 & $\ldots$ \\
\hline $\mathbf{Q} 2$ & 7,881 & 3,370 & 4,511 & 17,175 & 4,837 & 4,457 & 19,099 & 1,924 & $\ldots$ \\
\hline Q3 & 8,715 & 3,911 & 4,804 & 17,639 & 4,502 & 4,422 & 19,609 & 1,970 & $\ldots$ \\
\hline Q4 & 10,508 & 4,786 & 5,722 & 17,852 & 3,772 & 3,572 & 45,634 & 2,570 & $\ldots$ \\
\hline 1995 Jamuary & 9,258 & 4,069 & 5,189 & 17,267 & 4,361 & 3,648 & 44,958 & 2,546 & $\ldots$ \\
\hline Febnuary & 9,405 & 3,936 & 5,469 & 17,000 & 4,403 & 3,192 & 44,837 & 2,488 & $\ldots$ \\
\hline March & 9,667 & 4,241 & 5,426 & 16,991 & 4,028 & 3,296 & 44,026 & 2,403 & $\ldots$ \\
\hline April & 10,410 & 4,688 & 5,722 & 17,814 & 4,010 & 3,394 & 44,944 & 2,387 & $\ldots$ \\
\hline May & 10,431 & 4,465 & 5,966 & 17,499 & 4,189 & 2,879 & 21,065 & 2,424 & 1,142 \\
\hline June & 10,588 & 4,713 & 5,875 & 17,331 & 4,053 & 2,690 & 20,866 & 2,421 & 1,114 \\
\hline July & 11,373 & 5,062 & 6,311 & 18,354 & 3,890 & 3,091 & 21,912 & 2,420 & 1,138 \\
\hline August & 11,826 & 4,928 & 6,898 & 19,032 & 3,828 & 3,378 & 22,520 & 2,411 & 1,077 \\
\hline September & 11,458 & 4,856 & 6,602 & 18,388 & 3,880 & 3,050 & 21,917 & 2,422 & 1,107 \\
\hline October & 11,922 & 4,790 & 7,132 & 18,525 & 3,836 & 2,767 & 22,219 & 2,400 & 1,294 \\
\hline November & 11,950 & 5,017 & 6,933 & 18,090 & 3,391 & 2,749 & 21,798 & 2,459 & 1,249 \\
\hline December & 12,533 & 5,965 & 6,568 & 18,785 & 3,187 & 3,065 & 22,654 & 2,701 & 1,168 \\
\hline 1996 January & 11,990 & 5,163 & 6,827 & 18,455 & 3,368 & 3,097 & 22,213 & 2,627 & 1,131 \\
\hline February & 11,582 & 5,134 & 6,448 & 18,248 & 3,610 & 3,056 & 22,064 & 2,669 & 1,147 \\
\hline March & 11,321 & 5,182 & 6,139 & 17,675 & 3,682 & 2,672 & 21,393 & 2,624 & 1,094 \\
\hline April & 11,970 & 5,818 & 6,152 & 18,125 & 3,458 & 2,697 & 22,016 & 2,612 & 1,279 \\
\hline May & 11,400 & 5,361 & 6,039 & 17,701 & 3,511 & 2,790 & 21,665 & 2,616 & 1,348 \\
\hline June & 11,568 & 5,445 & 6,123 & 17,976 & 3,575 & 2,833 & 21,747 & 2,607 & 1,164 \\
\hline July & 11,762 & 5,499 & 6,263 & 18,137 & 3,486 & 2,889 & 21,879 & 2,588 & 1,154 \\
\hline August & 12,277 & 5,501 & 6,776 & 18,735 & 3,570 & 2,888 & 22,730 & 2,665 & 1,330 \\
\hline September & 12,038 & 5,500 & 6,538 & 18,341 & 3,491 & 2,812 & 22,299 & 2,689 & 1,269 \\
\hline October & 12,349 & 5,591 & 6,758 & 18,615 & 3,515 & 2,751 & 22,336 & 2,649 & 1,072 \\
\hline November & 11,698 & 5,741 & 5,957 & 18,363 & 3,783 & 2,882 & 22,055 & 2,733 & 959 \\
\hline December & 12,143 & 6,698 & 5,445 & 18,502 & 3,641 & 2,718 & 22,451 & 2,819 & 1,130 \\
\hline
\end{tabular}


Table 21. FYRM: Summary of General Government 1/

\begin{tabular}{|c|c|c|c|c|c|c|c|c|c|c|}
\hline & 1992 & 1993 & 1994 & 1995 & 199621 & 1992 & 1993 & 1994 & 1995 & 1996 \\
\hline & \multicolumn{5}{|c|}{ (In millions of denars) } & \multicolumn{5}{|c|}{ (In percent of GDP) } \\
\hline Total revenue and grants & 4,639 & 23,767 & 63,157 & 64,254 & 64,445 & 38.6 & 40.9 & 51.0 & 45.3 & 43.8 \\
\hline Central government & 2,238 & 13,411 & 37,993 & 40,874 & 40,189 & 18.6 & 23.1 & 30.7 & 28.8 & 27.3 \\
\hline Tax revenue & 2,187 & 11,453 & 35,492 & 37,261 & 37,715 & 18.2 & 19.7 & 28.7 & 26.2 & 25.7 \\
\hline Non-tax revenue & 45 & 1,657 & 2,501 & 2,829 & 2,369 & 0.4 & 2.8 & 2.0 & 2.0 & 1.6 \\
\hline Capital revenue & 6 & 301 & o & 122 & 6 & 0.0 & 0.5 & 0.0 & 0.1 & 0.0 \\
\hline Foreign grants & 0 & 0 & 0 & 662 & 99 & 0.0 & 0.0 & 0.0 & 0.5 & 0.1 \\
\hline Extrabudetary funds & 2,376 & 10,176 & 24,710 & 22,757 & 23,620 & 19.8 & 17.5 & 20.0 & 16.0 & 16.1 \\
\hline Pencion finds & 1,116 & 6,115 & 14,441 & 14,971 & 15,142 & 9.3 & 10.5 & 11.7 & 10.5 & 10.3 \\
\hline Health - care find & 683 & 2,398 & 6,032 & 6,117 & 6,662 & 5.7 & 4.1 & 4.9 & 4.3 & 4.5 \\
\hline Employment fund & 57 & 342 & 952 & 964 & 930 & 0.5 & 0.6 & 0.8 & 0.7 & 0.6 \\
\hline Other find & 520 & 1,320 & 3,286 & 704 & 886 & 4.3 & 2.3 & 2.7 & 0.5 & 0.6 \\
\hline Local buidgets & 25 & 180 & 453 & 623 & 636 & 0.2 & 0.3 & 0.4 & 0.4 & 0.4 \\
\hline Total expenditure and net lendings & 5,791 & 31,690 & 67,061 & 66,032 & 65,096 & 48.2 & 54.5 & 54.2 & 46.5 & 44.3 \\
\hline Central government & 3,134 & 17,658 & 36,843 & 35,745 & 33,774 & 26.1 & 30.4 & 29.8 & 25.2 & 23.0 \\
\hline Curreat expenditures & 3,020 & 17,081 & 33,187 & 32,461 & 30,458 & 25.2 & 29.4 & 26.8 & 22.9 & 20.7 \\
\hline Cepital expenditures & 100 & 527 & 3,466 & 3,133 & 3,221 & 0.8 & 0.9 & 2.8 & 2.2 & 2.2 \\
\hline Reserves & 14 & 49 & 190 & 151 & 95 & 0.1 & 0.1 & 0.2 & 0.1 & 0.1 \\
\hline Extrabudgetery finds & 2,628 & 13,852 & 29,765 & 29,703 & 30,688 & 21.9 & 23.8 & 24.0 & 20.9 & 20.9 \\
\hline Pension funds & 1,337 & 8,513 & 16,505 & 16,238 & 16,632 & 11.1 & 14.6 & 13.3 & 11.4 & 11.3 \\
\hline Health - care fund & 702 & 3,556 & 7,992 & 9,114 & 9,699 & 5.8 & 6.1 & 6.5 & 6.4 & 6.6 \\
\hline Employment find & 69 & 464 & 734 & 1,575 & 2,184 & 0.6 & 0.8 & 0.6 & 1.1 & 1.5 \\
\hline Other find & 520 & 1,320 & 4,534 & 2,776 & 2,173 & 4.3 & 2.3 & 3.7 & 2.0 & 1.5 \\
\hline Local Budgets & 30 & 180 & 453 & 585 & 635 & 0.2 & 0.3 & 0.4 & 0.4 & 0.4 \\
\hline \multicolumn{11}{|l|}{ Deficit (ccervals) } \\
\hline Net of transfers & $-1,152$ & $-7,923$ & $-3,904$ & $-1,778$ & -652 & -9.6 & -13.6 & -3.2 & -1.3 & -0.4 \\
\hline Central government & -896 & $-4,247$ & 1,150 & 5,129 & 6,415 & -7.5 & -7.3 & 0.9 & 3.6 & 4.4 \\
\hline Extrabudgetery funds & -252 & $-3,676$ & $-5,055$ & $-6,946$ & $-7,068$ & -2.1 & -6.3 & -4.1 & -4.9 & -4.8 \\
\hline Pension funds & -221 & $-2,397$ & $-2,064$ & $-1,267$ & $-1,490$ & -1.8 & -4.1 & -1.7 & -0.9 & -1.0 \\
\hline Health - care fund & -19 & $-1,157$ & $-1,960$ & $-2,997$ & $-3,037$ & -0.2 & -2.0 & -1.6 & -2.1 & -2.1 \\
\hline Employment fund & -12 & -122 & 218 & -611 & $-1,254$ & -0.1 & -0.2 & 0.2 & -0.4 & -0.9 \\
\hline Other fund & $\mathbf{0}$ & $\mathbf{0}$ & $-1,249$ & $-2,072$ & $-1,287$ & 0.0 & 0.0 & -1.0 & -1.5 & -0.9 \\
\hline Local budgets & -5 & $\mathbf{0}$ & o & 38 & 1 & 0.0 & 0.0 & 0.0 & 0.0 & 0.0 \\
\hline
\end{tabular}

Sources: Ministry of Finance; and staff estimates.

1/ Excludes transfers within the general government.

2/ Staff estimates and projections.

CInternational Monetary Fund. Not for Redistribution 
Table 22. FYRM: General Government Revenue and Grants $1 /$

\begin{tabular}{|c|c|c|c|c|c|c|c|c|c|c|}
\hline & 1992 & 1993 & 1994 & 1995 & $19962 /$ & 1992 & 1993 & 1994 & 1995 & 199621 \\
\hline & \multicolumn{5}{|c|}{ (In millions of denars) } & \multicolumn{5}{|c|}{ (In percent of GDP) } \\
\hline Total revenue and grants & 4,639 & 23,767 & 63,157 & 64,254 & 64,445 & 38.6 & 40.9 & 51.0 & 45.3 & 43.8 \\
\hline Total revenue (current and capital) & $\ldots$ & $\ldots$ & 63,157 & 63,592 & 64,346 & $\ldots$ & ... & 51.0 & 44.8 & 43.8 \\
\hline Current revenue (Tax and nontax) & $\ldots$ & $\ldots$ & 63,157 & 63,470 & 63,700 & $\ldots$ & $\ldots$ & 51.0 & 44.7 & 43.3 \\
\hline Tax revenue & $\ldots$ & $\ldots$ & 57,049 & 59,472 & 59,688 & $\ldots$ & $\ldots$ & 46.1 & 41.9 & 40.6 \\
\hline Taxes on income and profits & $\ldots$ & $\ldots$ & 11,520 & 11,098 & 11,191 & $\ldots$ & $\ldots$ & 9.3 & 7.8 & 7.6 \\
\hline Social security contribution & $\ldots$ & $\ldots$ & 20,467 & 20,954 & 20,785 & $\ldots$ & $\ldots$ & 16.5 & 14.8 & 14.1 \\
\hline Taxes on property & $\ldots$ & $\cdots$ & 255 & 402 & 411 & $\ldots$ & $\ldots$ & 0.2 & 0.3 & 0.3 \\
\hline Domestic taxes on goods and services & $\ldots$ & $\ldots$ & 19,029 & 20,189 & 20,654 & $\ldots$ & $\ldots$ & 15.4 & 14.2 & 14.1 \\
\hline Sales tax / VAT & $\ldots$ & $\ldots$ & 6,963 & 7,899 & 8,777 & $\ldots$ & $\ldots$ & 5.6 & 5.6 & 6.0 \\
\hline Excises & $\ldots$ & $\ldots$ & 12,066 & 12,290 & 11,877 & $\ldots$ & $\ldots$ & 9.7 & 8.7 & 8.1 \\
\hline Taxes on intemational trade and transactions & $\ldots$ & $\ldots$ & 5,778 & 6,829 & 6,647 & $\ldots$ & $\ldots$ & 4.7 & 4.8 & 4.5 \\
\hline Non tax revenue & $\ldots$ & $\ldots$ & 6,108 & 3,997 & 4,012 & $\ldots$ & $\ldots$ & 4.9 & 2.8 & 2.7 \\
\hline Capital revenue & $\ldots$ & $\ldots$ & 0 & 122 & 646 & $\ldots$ & $\ldots$ & 0.0 & 0.1 & 0.4 \\
\hline Foreign grants & $\ldots$ & $\ldots$ & $\mathbf{0}$ & 662 & 99 & $\ldots$ & $\ldots$ & 0.0 & 0.5 & 0.1 \\
\hline
\end{tabular}

Sources: Ministry of Finance; and staff estimates.

1/ Excludes transfers within the goneral government.

2/ Staff estimates and projections. 
Table 23. FYRM: General Government Expenditures 1/

\begin{tabular}{|c|c|c|c|c|c|c|c|c|c|c|}
\hline & 1992 & 1993 & 1994 & 1995 & 199621 & 1992 & 1993 & 1994 & 1995 & $19962 /$ \\
\hline & \multicolumn{5}{|c|}{ (In millions of denars) } & \multicolumn{5}{|c|}{ (In percent of GDP) } \\
\hline Total expenditure and net lending & 5,791 & 31,690 & 67,062 & 66,032 & 65,096 & 48.2 & 54.5 & 54.2 & 46.5 & 44.3 \\
\hline Current expenditure & $\ldots$ & $\ldots$ & 62,348 & 61,208 & 60,758 & $\ldots$ & $\ldots$ & 50.4 & 43.1 & 41.3 \\
\hline Wages and salaries & $\ldots$ & $\ldots$ & 14,201 & 14,965 & 15,629 & $\ldots$ & $\ldots$ & 11.5 & 10.5 & 10.6 \\
\hline Other purchase of goods and services & $\ldots$ & $\ldots$ & 5,516 & 6,969 & 5,419 & $\ldots$ & $\ldots$ & 4.5 & 4.9 & 3.7 \\
\hline Interest & $\ldots$ & $\ldots$ & 5,161 & 2,783 & 3,509 & $\ldots$ & $\ldots$ & 4.2 & 2.0 & 2.4 \\
\hline Domestic & $\ldots$ & $\ldots$ & 2,055 & 1,978 & 2,030 & $\ldots$ & $\ldots$ & 1.7 & 1.4 & 1.4 \\
\hline Foneign & ... & $\ldots$ & 3,106 & 805 & 1,479 & $\ldots$ & $\ldots$ & 2.5 & 0.6 & 1.0 \\
\hline Subsidies & $\ldots$ & $\ldots$ & 4,235 & 4,080 & 3,205 & $\ldots$ & $\ldots$ & 3.4 & 2.9 & 2.2 \\
\hline Unemployment related benefits & $\ldots$ & $\ldots$ & 618 & 1,459 & 2,049 & $\ldots$ & $\ldots$ & 0.5 & 1.0 & 1.4 \\
\hline Social assistance & $\ldots$ & $\ldots$ & 4,067 & 4,876 & 3,584 & $\ldots$ & $\ldots$ & 3.3 & 3.4 & 2.4 \\
\hline Pensions & $\ldots$ & $\ldots$ & 15,962 & 15,935 & 16,330 & $\ldots$ & $\ldots$ & 12.9 & 11.2 & 11.1 \\
\hline Health & $\ldots$ & $\ldots$ & 7,767 & 8,795 & 9,412 & $\ldots$ & $\ldots$ & 6.3 & 6.2 & 6.4 \\
\hline Other current & $\ldots$ & $\ldots$ & 4,821 & 1,347 & 1,622 & $\ldots$ & $\ldots$ & 3.9 & 0.9 & 1.1 \\
\hline Capital expenditure & $\ldots$ & $\ldots$ & 4,714 & 4,824 & 4,338 & $\ldots$ & $\ldots$ & 3.8 & 3.4 & 3.0 \\
\hline
\end{tabular}

Sources: Ministry of Finance; and staff estimates.

1/ Excludes transfers within the General Government.

$2 /$ Staff estimates and projections. 
Table 24: FYRM: Exchange Rate Developments 1/

(period averages)

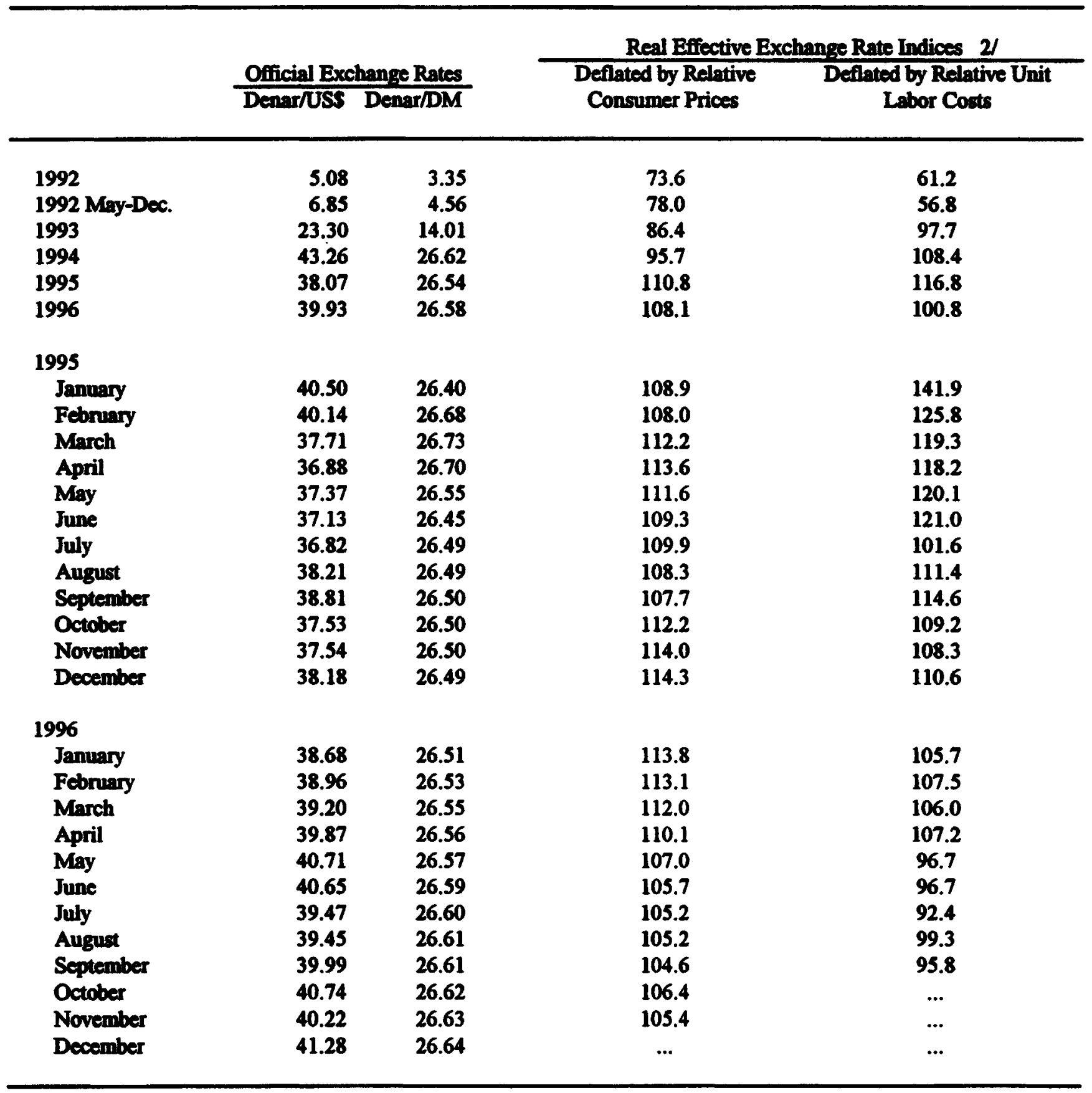

Sources: Data provided by the authorities; and staff estimates.

1/ The former SFRY dinar was the official currency prior to monetary independence on April 26, 1992, when the official currency became the FYRM denar. In May 1993, a currency reform took place with the introduction of a new denar equal to 100 old denars. All exchange rates shown are expressed in terms of the new denar.

2/ Staff extimates. December 1992=100. An increase in the index indicates an appreciation. 
Table 25. FYRM: Balance of Payments

(In millions of U.S. dollars)

\begin{tabular}{|c|c|c|c|c|c|}
\hline & 1992 & $19931 /$ & 1994 & $\frac{\text { Rev }}{1995}$ & $\frac{\text { Est. }}{1996}$ \\
\hline Current account & -19 & -87 & -181 & .232 & -437 \\
\hline Trade balance $2 /$ & -7 & -171 & -186 & -235 & -523 \\
\hline Exports & 1,199 & 1,056 & 1,086 & 1,204 & 912 \\
\hline Imports (including aid) & 1,206 & 1,227 & 1,272 & 1,439 & 1,435 \\
\hline Income, net & -72 & -75 & -62 & -38 & -9 \\
\hline $\begin{array}{l}\text { Services and tranafers, } \\
\text { net (excluding interest) }\end{array}$ & 60 & 159 & 67 & 41 & 95 \\
\hline Capital sccount & -96 & -19 & 18 & 53 & 143 \\
\hline Public sector & -96 & -2 & 64 & 16 & 42 \\
\hline Disbursements & 9 & 57 & 149 & 103 & 116 \\
\hline Amortization & 105 & -59 & 84 & 87 & 74 \\
\hline Private sector & $\mathbf{0}$ & -17 & -46 & 36 & 101 \\
\hline Direct/portfolio investment & 0 & 0 & 24 & 13 & 39 \\
\hline Commercial banks' position, net & 0 & .17 & -70 & 23 & 62 \\
\hline Errora/omissions and short-term capital 3/ & 21 & 94 & 204 & 165 & 238 \\
\hline Overall balance & .94 & -12 & 41 & -15 & -56 \\
\hline Financing & 94 & 12 & -41 & 15 & 56 \\
\hline Change in grows foreign assets (increase: -) & -61 & -60 & -46 & -101 & 1 \\
\hline MF (net change) & 0 & 0 & 16 & 37 & 14 \\
\hline Change in arrears & 155 & 72 & -11 & -247 & 8 \\
\hline New arrears & 155 & 72 & 100 & 71 & 32 \\
\hline Repayment of arrears & 0 & 0 & 111 & 318 & 24 \\
\hline Rescheduling & 0 & 0 & 0 & 326 & 33 \\
\hline \multicolumn{6}{|l|}{ Memorandum items: } \\
\hline (in percent of GDP) & -.8 & -3.5 & -6.3 & -6.2 & -11.9 \\
\hline $\begin{array}{l}\text { Gross reserves } \\
\text { (in months of reoular imports) }\end{array}$ & & & & & \\
\hline (in months of regular imports) & 0.6 & 1.2 & 1.3 & 1.9 & 2.0 \\
\hline
\end{tabular}

Sources: The authorities; and staff estimates.

1/ From 1993 forward, figures incorponate claceification, and mothodologioul changes es rocommended by the Statistics Department.

$2 /$ Changes in the coverage of trade data during the period result in discontinuities in trade series, and related ratios, which are particularly evident in 1996.

3/ Includes unidentified short-term capital, such as trade credits, imports paid by drawndown of foreign held bank accounts, and higher than recorded inflows of remittances. 
Table 26. FYRM: Commodity Composition of Exports 1/

(In percent of total exports)

\begin{tabular}{|c|c|c|c|c|c|c|}
\hline & 1990 & 1991 & 1992 & 1993 & 1994 & 1995 \\
\hline Exports & 100 & 100 & 100 & 100 & 100 & 100 \\
\hline Food and live animals & 3 & 5 & 15 & 11 & 10 & 11 \\
\hline Beverages and tobacco & 3 & 7 & 12 & 10 & 6 & 7 \\
\hline Crude materials, except fuels & 5 & 5 & 5 & 6 & 7 & 8 \\
\hline Chemicals & 6 & 3 & 4 & 4 & 5 & 6 \\
\hline \multicolumn{7}{|l|}{ Manufactured goods } \\
\hline classified by materials & 47 & 42 & 30 & 34 & 35 & 35 \\
\hline Machinery and transport equipment & 9 & 10 & 8 & 16 & 12 & 13 \\
\hline Miscellaneous manufactured goods & 27 & 23 & 25 & 18 & 22 & 18 \\
\hline Other & 0 & 5 & 1 & 1 & 3 & 2 \\
\hline
\end{tabular}

Source: Data provided by the authorities.

1/ Including, from 1991 onwards, exports to the republics of the former SFRY. 
Table 27. FYRM: Destination of Exports

(In percent of total exports)

\begin{tabular}{|c|c|c|c|c|c|c|}
\hline & 1991 & 1992 & 1993 & 1994 & 1995 & 1996 \\
\hline Total exports & 100 & 100 & 100 & 100 & 100 & 100 \\
\hline Republics of former SFRY 1/ & 5 & 22 & 19 & 13 & 16 & 41 \\
\hline $\begin{array}{l}\text { Former CMEA area } \\
\text { Of which: }\end{array}$ & 33 & 19 & 27 & 35 & 36 & 14 \\
\hline Russia & 23 & 8 & 11 & 7 & 7 & 4 \\
\hline Bulgaris 2/ & 4 & 5 & 8 & 21 & 21 & 4 \\
\hline Czech Republic & 3 & 2 & 2 & 1 & 1 & 1 \\
\hline Albania & 0.5 & 2 & 3 & 3 & 2 & 4 \\
\hline $\begin{array}{l}\text { European Union } \\
\text { Of which: }\end{array}$ & 43 & 42 & 35 & 32 & 34 & 34 \\
\hline Germany & 21 & 20 & 14 & 13 & 13 & 13 \\
\hline Italy & 7 & 8 & 9 & 11 & 10 & 5 \\
\hline Greece & 1 & 2 & 2 & 2 & 3 & 2 \\
\hline United Kingdom & 6 & 3 & 5 & 1 & 1 & 7 \\
\hline Austria & 2 & 2 & 1 & 1 & 2 & 3 \\
\hline $\begin{array}{l}\text { Other } \\
\text { Of which: }\end{array}$ & 19 & 17 & 19 & 20 & 14 & 11 \\
\hline U.S.A. & 4 & 6 & 6 & 4 & 3 & 4 \\
\hline Switzerland & 2 & 3 & 3 & 3 & 3 & 3 \\
\hline Turkey & 2 & 2 & 4 & 3 & 3 & 2 \\
\hline
\end{tabular}

Source: Data provided by the authorities.

1/ Exports to the republics of the former SFRY are only recorded fully as external trade from 1992.

2/ A large part of the increase in exports to Bulgaria in 1993-95 is believed to reflect increased transit of exports. 
Table 28. FYRM: Exports and Imports by Final Use

(In percent of total)

\begin{tabular}{lrrrrrr}
\hline & 1992 & 1992 & 1993 & 1995 & $\frac{\text { Seml }}{1996}$ \\
\hline $\begin{array}{l}\text { Exports } \\
\text { Industrial supplies }\end{array}$ & 100 & 100 & 100 & 100 & 100 & 100 \\
$\begin{array}{l}\text { Investment goods and } \\
\text { spare parts }\end{array}$ & 58 & 43 & 48 & 50 & 54 & 54 \\
$\begin{array}{l}\text { Consumption goods } \\
\text { Not specified }\end{array}$ & 6 & 3 & 7 & 5 & 4 & 4 \\
$\begin{array}{l}\text { Imports } \\
\text { Industrial supplies } \\
\text { of which: oil and } \\
\text { derivatives }\end{array}$ & 36 & 51 & 40 & 41 & 37 & 38 \\
$\begin{array}{l}\text { Investment goods and } \\
\text { spare parts }\end{array}$ & 100 & 3 & 5 & 4 & 5 & 4 \\
$\begin{array}{c}\text { Consumption goods } \\
\text { Not specified }\end{array}$ & 70 & 100 & 100 & 100 & 100 & 100 \\
\hline & 11 & 11 & 15 & 10 & 11 & 10 \\
\hline
\end{tabular}

Source: Data provided by the authorities. 
Table 29. FYRM: Commodity Composition of Imports 1/

(In percent of total imports)

\begin{tabular}{|c|c|c|c|c|c|c|}
\hline & 1990 & 1991 & 1992 & 1993 & 1994 & 1995 \\
\hline Imports & 100 & 100 & 100 & 100 & 100 & 100 \\
\hline Food and live animals & 10 & 9 & 14 & 17 & 19 & 16 \\
\hline Beverages and tobacco & 3 & 3 & 2 & 2 & 1 & 1 \\
\hline Crude materials, except fuels & 8 & 7 & 9 & 6 & 5 & 5 \\
\hline \multicolumn{7}{|l|}{ Mineral fuels, lubricants } \\
\hline and related materials & 14 & 11 & 12 & 16 & 11 & 12 \\
\hline Animal and vegetable oils & 0 & 0 & 1 & 0 & $\mathbf{0}$ & 0 \\
\hline Chemicals & 9 & 9 & 13 & 15 & 13 & 12 \\
\hline \multicolumn{7}{|l|}{ Manufactured goods classified } \\
\hline by materials & 19 & 19 & 17 & 17 & 13 & 16 \\
\hline Machinery and transport equipment & 11 & 12 & 10 & 17 & 19 & 19 \\
\hline Miscellaneous manufactured goods & 10 & 9 & 10 & 7 & 9 & 9 \\
\hline Other 21 & 16 & 21 & 12 & 3 & 10 & 10 \\
\hline
\end{tabular}

Source: Data provided by the authorities.

1/ Including, from 1991 onwards, imports from the republics of the former SFRY. Excluding imports for humanitarien aid. 2/ In 1991, approximately 7 percentage points of "Other" imports represent imports from the republics of the former SFRY as no break-down by commodity is available for these imports in this year. 
Table 30. FYRM: Origin of Imports

(In percent of total imports)

\begin{tabular}{|c|c|c|c|c|c|c|}
\hline & 1991 & 1992 & 1993 & 1994 & 1995 & 1996 \\
\hline Total exports & 100 & 100 & 100 & 100 & 100 & 100 \\
\hline Republics of the former SFRY $1 /$ & 7 & 13 & 24 & 21 & 19 & 21 \\
\hline $\begin{array}{l}\text { Former CMEA area } \\
\text { Of which: }\end{array}$ & 39 & 34 & 28 & 25 & 25 & 21 \\
\hline Russia & 27 & 14 & 12 & 3 & 4 & 8 \\
\hline Bulgaria 2/ & 5 & 14 & 11 & 16 & 15 & 7 \\
\hline Czech Republic & 3 & 2 & 1 & 2 & 1 & 1 \\
\hline Albania & 1 & 2 & 3 & 3 & 2 & 4 \\
\hline $\begin{array}{l}\text { European Union } \\
\text { Of which: }\end{array}$ & 41 & 38 & 33 & 36 & 40 & 39 \\
\hline Germany & 17 & 14 & 13 & 17 & 17 & 15 \\
\hline Italy & 6 & 10 & 6 & 7 & 10 & 7 \\
\hline Greece & 4 & 2 & 2 & 3 & 3 & 2 \\
\hline United Kingdom & 7 & 4 & 4 & 2 & 2 & 5 \\
\hline Austria & 2 & 1 & 1 & 1 & 1 & 1 \\
\hline $\begin{array}{l}\text { Other } \\
\text { Of which: }\end{array}$ & 13 & 15 & 15 & 18 & 16 & 19 \\
\hline U.S.A. & 2 & 2 & 2 & 3 & 3 & 4 \\
\hline Switzerland & 2 & 1 & 2 & 2 & 1 & 2 \\
\hline Turkey & 2 & 3 & 3 & 3 & 3 & 3 \\
\hline
\end{tabular}

Source: Data provided by the authorities.

1/ Imports from the republics of the former SFRY only began to be recorded fully as external trade from 1992.

2/ A large part of the increase in imports to Bulgaria in 1992-95 is believed to reflect increased transit of imports, however, break-down is not available of transit imports and final imports to Bulgaria. 
Table 31. FYRM: Services Account

(In millions of U.S. dollars)

\begin{tabular}{|c|c|c|c|c|c|}
\hline & 1992 & 1993 & 1994 & 1995 & 1996 \\
\hline $\begin{array}{l}\text { Travel, net } \\
\text { Receipts } \\
\text { Payments }\end{array}$ & $\begin{array}{r}4 \\
11 \\
-7\end{array}$ & $\begin{array}{r}0 \\
13 \\
-13\end{array}$ & $\begin{array}{r}6 \\
29 \\
-23\end{array}$ & $\begin{array}{r}-8 \\
19 \\
-27\end{array}$ & $\begin{array}{r}-6 \\
20 \\
-26\end{array}$ \\
\hline $\begin{array}{l}\text { Transportation, net } \\
\text { Receipts } \\
\text { Payments }\end{array}$ & $\begin{array}{r}-6 \\
6 \\
-12\end{array}$ & $\begin{array}{r}-115 \\
23 \\
-138\end{array}$ & $\begin{array}{r}-127 \\
39 \\
-166\end{array}$ & $\begin{array}{r}-138 \\
54 \\
-192\end{array}$ & $\begin{array}{r}-102 \\
51 \\
-153\end{array}$ \\
\hline $\begin{array}{l}\text { Other, net } \\
\text { Receipts } \\
\text { Payments }\end{array}$ & $\begin{array}{r}32 \\
43 \\
-11\end{array}$ & $\begin{array}{r}-39 \\
48 \\
-87\end{array}$ & $\begin{array}{r}-35 \\
104 \\
-139\end{array}$ & $\begin{array}{r}-41 \\
81 \\
-122\end{array}$ & $\begin{array}{r}-33 \\
95 \\
-129\end{array}$ \\
\hline $\begin{array}{l}\text { Total services } \\
\text { excluding interest, net } \\
\text { Receipts } \\
\text { Payments }\end{array}$ & $\begin{array}{r}30 \\
60 \\
-30\end{array}$ & $\begin{array}{r}-154 \\
84 \\
-238\end{array}$ & $\begin{array}{r}-156 \\
172 \\
-328\end{array}$ & $\begin{array}{r}-187 \\
155 \\
-341\end{array}$ & $\begin{array}{r}-141 \\
167 \\
-308\end{array}$ \\
\hline $\begin{array}{l}\text { Income, net } \\
\text { Receipts } \\
\text { Payments }\end{array}$ & $\begin{array}{r}-72 \\
0 \\
-72\end{array}$ & $\begin{array}{r}-56 \\
5 \\
-61\end{array}$ & $\begin{array}{r}-63 \\
9 \\
-72\end{array}$ & $\begin{array}{r}-38 \\
20 \\
-58\end{array}$ & $\begin{array}{r}-9 \\
44 \\
-53\end{array}$ \\
\hline $\begin{array}{l}\text { Total services, net } \\
\text { Receipts } \\
\text { Payments }\end{array}$ & $\begin{array}{r}-42 \\
61 \\
-102\end{array}$ & $\begin{array}{r}-210 \\
89 \\
-299\end{array}$ & $\begin{array}{r}-219 \\
181 \\
-400\end{array}$ & $\begin{array}{r}-224 \\
175 \\
-399\end{array}$ & $\begin{array}{r}-150 \\
211 \\
-361\end{array}$ \\
\hline
\end{tabular}

Sources: Data and estimates provided by the authorities; and staff estimates. 


\section{Table 32. FYRM: Official Gold and Convertible Foreign Exchange Reserves}

(In millions of U.S. dollars; end-of-period)

\begin{tabular}{|c|c|c|c|c|c|c|}
\hline & & Gold 1/ & $\begin{array}{c}\text { SDR } \\
\text { Holdings }\end{array}$ & $\begin{array}{l}\text { Reserve } \\
\text { Position } \\
\text { in the Fund }\end{array}$ & $\begin{array}{l}\text { Foreign } \\
\text { Exchange }\end{array}$ & Total \\
\hline 1993: & $\begin{array}{l}\text { Q1 } \\
\text { Q2 } \\
\text { Q3 } \\
\text { Q4 }\end{array}$ & $\begin{array}{r}7.1 \\
11.5 \\
13.8 \\
14.7\end{array}$ & $\begin{array}{l}0.2 \\
0.3 \\
0.2 \\
0.2\end{array}$ & $\begin{array}{l}- \\
- \\
-\end{array}$ & $\begin{array}{r}58.2 \\
96.4 \\
118.1 \\
104.6\end{array}$ & $\begin{array}{r}65.5 \\
108.2 \\
132.1 \\
119.5\end{array}$ \\
\hline 1994: & $\begin{array}{l}\text { Q1 } \\
\text { Q2 } \\
\text { Q3 } \\
\text { Q4 }\end{array}$ & $\begin{array}{l}14.7 \\
15.3 \\
16.4 \\
16.4\end{array}$ & $\begin{array}{l}0.6 \\
0.3 \\
1.0 \\
--\end{array}$ & $\begin{array}{l}- \\
- \\
-- \\
-\end{array}$ & $\begin{array}{r}88.7 \\
140.7 \\
146.6 \\
149.0\end{array}$ & $\begin{array}{l}104.0 \\
156.3 \\
163.9 \\
165.4\end{array}$ \\
\hline 1995: & $\begin{array}{l}\text { Q1 } \\
\text { Q2 } \\
\text { Q3 } \\
\text { Q4 }\end{array}$ & $\begin{array}{l}16.4 \\
16.4 \\
16.4 \\
17.6\end{array}$ & $\begin{array}{l}-- \\
-- \\
-- \\
-\end{array}$ & $\begin{array}{l}- \\
- \\
-\end{array}$ & $\begin{array}{l}162.4 \\
193.5 \\
223.3 \\
257.3\end{array}$ & $\begin{array}{l}178.9 \\
209.9 \\
239.7 \\
274.9\end{array}$ \\
\hline 1996: & $\begin{array}{l}\text { Q1 } \\
\text { Q2 } \\
\text { Q3 } \\
\text { Q4 }\end{array}$ & $\begin{array}{l}19.7 \\
22.2 \\
22.2 \\
22.2\end{array}$ & $\begin{array}{l}- \\
-- \\
-- \\
-\end{array}$ & $\begin{array}{l}- \\
- \\
- \\
-\end{array}$ & $\begin{array}{l}232.2 \\
237.6 \\
231.5 \\
249.0\end{array}$ & $\begin{array}{l}251.9 \\
259.8 \\
253.7 \\
271.2\end{array}$ \\
\hline
\end{tabular}

Sources: Data supplied by the authorities; and by the Treasurer's Department, IMF.

1/ At national valuation. 
Table 33. FYRM: Extemal Debt Stock 1/

(In millions of U.S. dollars)

\begin{tabular}{|c|c|c|c|c|c|c|}
\hline & 1991 & 1992 & 1993 & 1994 & 1995 & 1996 \\
\hline Total & 744 & 812 & 873 & 898 & 1115 & 1176 \\
\hline Multilateral creditors & 201 & 261 & 296 & 288 & 398 & 467 \\
\hline $\mathbf{M M F}$ & 0 & 0 & 15 & 33 & 69 & 80 \\
\hline IBRD/IDA & 108 & 161 & 175 & 131 & 178 & 206 \\
\hline IFC & 9 & 10 & 10 & 10 & 5 & 2 \\
\hline EIB & 52 & 58 & 59 & 70 & 82 & 76 \\
\hline Council of Europe & 6 & 7 & 7 & 8 & 10 & 6 \\
\hline EBRD & $\mathbf{0}$ & 0 & 0 & 1 & 15 & 69 \\
\hline EUROFIMA & 25 & 26 & 30 & 35 & 40 & 29 \\
\hline Bilateral creditors $2 /$ & 232 & 255 & 277 & 318 & 412 & 402 \\
\hline Commercial creditors & 311 & 296 & 300 & 293 & 305 & 307 \\
\hline Commercial banks & 283 & 283 & 283 & 283 & 283 & 283 \\
\hline Other & 28 & 13 & 17 & 10 & 22 & 24 \\
\hline Total not including IMF & 744 & 812 & 857 & 865 & 1046 & 1096 \\
\hline \multicolumn{7}{|l|}{ Memorandum items: } \\
\hline External debt (share of total) & 100.0 & 100.0 & 100.0 & 100.0 & 100.0 & 100.0 \\
\hline Multilateral creditors & 27.1 & 32.1 & 33.9 & 32.0 & 35.7 & 39.7 \\
\hline Bilateral creditors & 31.1 & 31.5 & 31.7 & 35.4 & 37.0 & 34.2 \\
\hline Commercial creditors & 41.8 & 36.4 & 34.4 & 32.6 & 27.4 & 26.1 \\
\hline External debt (as percent of GDP) & 15.6 & 34.4 & 34.8 & 31.4 & 29.9 & 32.1 \\
\hline
\end{tabular}

Sources: Data provided by authorities; and staff estimates.

1/Medium- and long-term debt only. Includes interest in arrears and late interest.

2/ Including debt guaranteed/insured by government/government agencies only. 